PALEO

Revue d'archéologie préhistorique

$28 \mid 2017$

Varia

\title{
Graver la pierre dans la vallée de la Vienne au Magdalénien moyen : le cas des chevaux sur supports mobiliers lithiques
}

Engraving rocks in the Vienne valley during Middle Magdalenian : the example

of horse figures on lithic portable art

Pascaline Gaussein

\section{OpenEdition}

Journals

Édition électronique

URL : http://journals.openedition.org/paleo/3323

DOI : $10.4000 /$ paleo.3323

ISSN : 2101-0420

Éditeur

SAMRA

Édition imprimée

Date de publication : 30 décembre 2017

Pagination : 137-156

ISSN : 1145-3370

Référence électronique

Pascaline Gaussein, "Graver la pierre dans la vallée de la Vienne au Magdalénien moyen : le cas des chevaux sur supports mobiliers lithiques », PALEO [En ligne], 28 | 2017, mis en ligne le 01 juin 2018, consulté le 10 décembre 2020. URL : http://journals.openedition.org/paleo/3323 ; DOI : https:// doi.org/10.4000/paleo.3323

\section{c) (i) $९($}

PALEO est mis à disposition selon les termes de la licence Creative Commons Attribution - Pas d'Utilisation Commerciale - Pas de Modification 4.0 International. 


\title{
Graver la pierre dans la vallée de la Vienne au Magdalénien moyen : le cas des chevaux sur supports mobiliers lithiques
}

\author{
Pascaline GAUSSEIN(a)
}

Résumé : Cet article fait état des résultats d'un Mémoire de recherche de Master 2 (Museum national d'Histoire naturelle, Paris, 2012). Les études passées ont montré que l'identité des groupes magdaléniens est fortement marquée entre les vallées de la Vienne et de la Charente, tant par leurs productions technoéconomiques que sociosymboliques. La présente étude vise à vérifier l'importance des conventions artistiques dans un tel contexte à travers l'analyse des pierres gravées. Le cheval, thème le plus fréquent de l'iconographie paléolithique, en constitue le point central (97 figures). Cette analyse repose sur un échantillon de 67 dalles, blocs, plaques et plaquettes gravées, mis au jour lors de fouilles anciennes ( $X X^{e}$ siècle et première moitié $d u X X^{\ominus}$ ) et récentes (années 1990 et 2000). Les cinq sites concernés se concentrent dans le département de la Vienne : La Marche, le Réseau Guy Martin et les Fadets (Lussac-les-Châteaux), le Taillis des Coteaux (Antigny) et la Grotte du Chaffaud (ou Puits au Chaffaud, Savigné). La relation entre le choix du support, les propriétés des surfaces ornées et les figurations animales et leur style a été analysée.

Cette étude a mis en évidence des constances et des variations de représentation au cours d'une même phase du Magdalénien ainsi qu'au sein d'un même site. Dans la quasi-totalité des cas étudiés, quelle que soit leur taille, ces supports dits « mobiles » n'ont finalement que rarement circulé, et leur caractère non périssable ne semble pas impliquer la pérennité des images ni leur qualité graphique. Certains chevaux gravés sont dynamiques, représentés avec de nombreux détails, dans des allures naturalistes, mais il existe également de nombreuses figures sommaires, « maladroites ", ou encore mal proportionnées, dont l'aspect ne dépend en rien de la qualité du support. Une contrainte ou un facteur non matériel entre donc en jeu. Au final, une cohérence de traditions graphiques apparaît sur les pierres gravées du Magdalénien moyen le long de la vallée de la Vienne, dont la variabilité de style et de facture des figures semble faire partie.

Mots-clés : Magdalénien moyen, Poitou, art mobilier, support lithique, conventions de représentation, cheval, analyse stylistique.

Abstract: Engraving rocks in the Vienne valley during Middle Magdalenian: the example of horse figures on lithic portable art. This article presents the main results of our Master thesis (National Museum of Natural History, Paris, 2012). Within the Magdalenian culture, despite of the wide spreading of certain weapons and raw materials, sociocultural practices and behaviours seem to be very specific, even exclusive to certain regions as the Vienne valley (West-Central France). The issue of this article is to discuss the strictness of representation in such a culturally coherent region. This study focused on the horse, main animal theme in the Magdalenian bestiary in this region. A hundred figures were analyzed, engraved on 67 stones of various sizes and geological natures, excaved from 5 main archaeological sites from the Vienne department: La Marche, Réseau Guy Martin and Les Fadets (Lussac-les-Châteaux), le Taillis des Coteaux (Antigny) and the Chaffaud cave (Savigné). The analysis focuses on the relation between the medium chosen to be engraved and its characteristics, and the animal figures depicted.

(a) Université Paris Nanterre, UMR 7055 Préhistoire et Technologie - pgaussein@hotmail.fr 
This work yields evidences of variations within the conventions of representation during the Middle Magdalenian, even within the same site. Most of those portable pieces are not destined to be spread, and these non-perishable medium do not imply that the images are neither carved to last nor to be works of art. Indeed, those images are often carved over with other figures and non-figurative incisions. Some animal figures are animated, detailed and represented in realistic and naturalistic postures, however numerous summary, distorted and inexpert figures have been carved, regardless of the proprieties and irregularities of the medium. Consequently, a non-medium related factor may have been responsible for those features. Eventually, the observed habits concerning the carved rocks show some graphic traditional cohesion, including the variability of style of the animal figures.

Key-words: Middle Magdalenian, Poitou, portable art, rock art, conventions of representation, horse, style analysis.

\section{Introduction}

Les conventions de représentation dans l'art paléolithique ne sont pas strictement homogènes sur toute l'extension d'un même technocomplexe : il existe des spécificités et des variations régionales, voire locales, notamment au cours du Magdalénien (Paillet 1998 ; Tosello 2003 ; Pigeaud 2007a ; Bourdier 2010a ; Rivero et Sauvet 2014). En effet, face à l'ampleur des réseaux de circulation des matières premières et de diffusion de certains outils et armatures du Magdalénien moyen (Langlais 2010 ; Primault 2012 ; Langlais et al. 2015), les vestiges de type socio-symboliques dessinent au contraire une zone culturelle restreinte dans le bassin versant de la Vienne (Airvaux 2000 ; Dujardin et Pinçon, 2000 ; Bourdier 2010b, 2010b). Une culture matérielle paraissant aussi bien définie et confinée impose-t-elle également des conventions stylistiques strictes, sans laisser place à la moindre variabilité ?

L'art gravé est le plus largement répandu au Paléolithique supérieur en France que ce soit sur supports mobiliers ou pariétaux. La région Poitou-Charentes compte des collections de pierres gravées quantitativement et qualitativement significatives pour mener cette réflexion. De plus, la figure du cheval, premier thème du bestiaire paléolithique (Leroi-Gourhan 1965) offre l'opportunité de travailler sur un échantillon d'objets représentatif (types de pièces et variété des figures) et appréhendable dans le cadre d'un mémoire de deuxième année de Master. Les pierres gravées de chevaux attribuées au Magdalénien sont exclusivement concentrées dans les sites du Seuil du Poitou et de son pourtour (fig. 1). De plus, les phases récentes du Magdalénien étant peu représentées (peu de sites et rares supports lithiques), nous nous concentrons donc ici sur le Magdalénien moyen autour de la vallée de la Vienne (67 pierres, 97 figures).

Ainsi, à partir de ce corpus d'étude, nous nous sommes interrogée sur le degré de variabilité des pratiques artistiques toléré dans le cadre d'une tradition culturelle régionale.

\section{1 - Un point sur le Magdalénien régional}

\section{1 - Contextualisation}

Dans la région étudiée, le Magdalénien moyen est globalement situé entre 15500 et 14000 BP (environ 19000 à 16500 ans cal BP IntCal 13 par OxCal 4.3 ; Dujardin et Pinçon 2000 ; Airvaux, Brou et Primault 2012). Il est contemporain de la fin du Pléniglaciaire supérieur et du début du Dryas ancien (ou Dryas I), période froide et aride caractérisée par un paysage principalement ouvert entre toundra steppique et prairies à graminées, et occupé par un cortège faunique arctique et steppique (Langlais 2010). Le renne est le principal taxon chassé dans le centre-ouest de la France étudié, suivi du cheval, dans des proportions variables selon les sites (Pinçon [Ed.] 2009 ; Primault et coll. 2010).

Au sud de la région considérée, le bassin versant de la Charente s'étend dans le nord du Périgord. À l'est et au nord : Vienne, Gartempe et Anglin font partie du vaste réseau hydrographique de la Loire.

Les approvisionnements en matières premières siliceuses et en coquillages suivent en bonne partie ces axes de circulation et d'installation des groupes préhistoriques, de sorte que le seuil du Poitou se situe bien au carrefour géographique et humain du Bassin parisien d'une part, et du Bassin aquitain d'autre part. Les fossiles et silex de Touraine (Grand-Pressigny, Fontmaure) sont retrouvés vers le sud jusque dans le Tarn-et-Garonne, et vers le nord jusqu'en Mayenne ; de même, le silex du bergeracois est exceptionnellement identifié dans la Vienne (Taborin 1993 ; Bourdier 2010b ; Langlais 2010).

La phase moyenne du Magdalénien connaît globalement une intensification de la production d'objets et une diversification des traditions techniques et stylistiques. Elle se caractérise par une production distincte des supports laminaires standardisés nécessitant des stocks de matière première de qualité, et des supports lamellaires plus opportunistes, et tend à se morceler spatialement par la production d'éléments d'armature, de parure et d'art régionaux, voire locaux (Langlais et al. 2015). II en ressort la distinction de traditions internes dites « faciès ", tels que les faciès de la Garenne (à navettes) et de Lussac-Angles (sagaies du même nom ; Allain et al. 1985 ; Paillet, Pinçon 


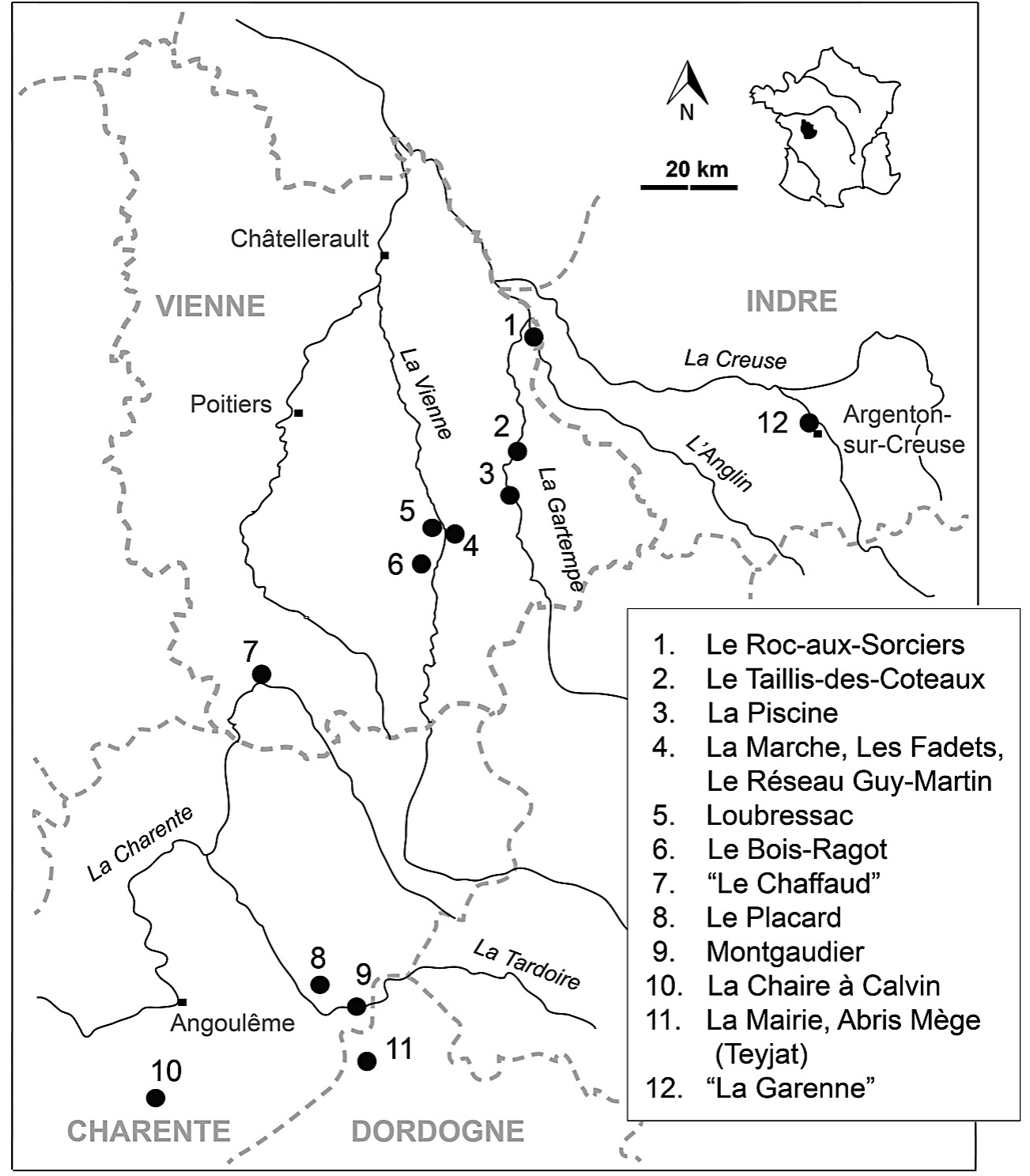

Figure 1 - Principaux sites magdaléniens de la Vienne et des départements alentours cités dans l'article. DAO P. Gaussein.

Figure 1 - Main Magdalenian sites of the Vienne and from surrounding departments mentioned in this article. CAD by P. Gaussein. et Bourdier 2016), ou encore à lamelles scalènes (Langlais et al. 2015).

\section{2 - Expressions graphiques du Magdalénien moyen régional}

Le Magdalénien moyen constitue la phase culturelle la mieux représentée et la plus riche en art mobilier lithique et pariétal. Les sites majeurs de la région sont l'abri du Rocaux-Sorciers à Angles-sur-l'Anglin, avec en particulier de l'art pariétal gravé, sculpté et peint, et la grotte de La Marche à Lussac-les-Châteaux, dont l'art se compose presque essentiellement de supports mobiliers lithiques gravés et dont l'estimation s'élève à près de 3000 pièces et fragments de pièces ${ }^{1,2}$ (fig. 1). II faut également noter à Lussac-les-Châteaux l'existence d'un art pariétal et mobilier lithique gravé au réseau Guy Martin³ (Airvaux 1998, 2001, 2011), ainsi que 169 pierres gravées à la grotte des Fadets ${ }^{4}$ (Airvaux 2001), et enfin des collections d'art mobilier en

(1) Le Roc-aux-Sorciers niv. C1: $14770 \pm 140$ BP (GifA-94190) ; niv. C3/D : $14510 \pm 160$ BP (GifA-94191) ; niv. D : $14160 \pm 80$ BP (GRN 1913) (Pinçon [Ed.] 2009).

(2) La Marche niv. unique : $14280 \pm 160$ BP (Ly 21000 ; Airvaux 2001).

(3) Le réseau Guy Martin niv. unique : $14240 \pm 85$ BP (Orsay 3780 ; Airvaux 2001).

(4) Les Fadets niv. inconnu : $15300 \pm 50$ BP (Gif 7353 ; Airvaux 2001). 
bien moindres proportions au Taillis des Coteaux à Antigny ${ }^{5}$ (Airvaux 2006 ; Primault et coll. 2010 ; Gaussein 2012), et

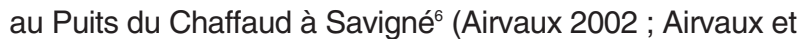
Leuvrey 2009, Gaussein 2014). De manière générale, il apparaît une nette dominance des supports lithiques sur les supports osseux.

Dans la région étudiée, deux principaux faciès culturels ont été identifiés. Le faciès dit " de Lussac-Angles » est caractérisé par une expression graphique strictement régionale, et notamment par la gravure d'incisives de poulains, les perles « stomach-bead", et un grand nombre de représentations animales et humaines naturalistes et réalistes en pied ou sous forme de portraits (Pales et Tassin de Saint Péreuse 1981 ; Airvaux 2000, 2001, 2011 ; Bourdier 2010a, 2010b ; Fuentes 2013a, 2013b ; Fuentes et al. 2016 ; Paillet, Pinçon et Bourdier 2016). Ce faciès est observé au Roc-aux-Sorciers, aux Fadets, au Chaffaud (Savigné), à la Piscine (Montmorillon) et à La Marche, et peut-être au Taillis des Coteaux (fig. 1 ; Airvaux 2002 ; Pinçon et Bertrand 2009 ; Primault et coll. 2010 ; Delage 2011 ; Airvaux, Brou et Primault 2012 ; Gaussein 2013 ; Paillet, Pinçon et Bourdier 2016).

Au contraire, le Magdalénien à « navettes » ou « de type La Garenne » (du site éponyme, Saint-Marcel, Indre) présente plutôt des images abstraites et de rares figures humaines à tendance schématique pour lesquelles il est bien connu (Allain et al. 1985 ; Fuentes 2013a, 2013b ; Fuentes et al. op. cit. ; Paillet, Pinçon et Bourdier op. cit.). Des figures animales moins schématiques ont cependant été identifiées sur des supports mobiliers lithiques (Paillet 2009). Ce faciès serait également représenté au Chaffaud et à la Piscine, moins assurément au Taillis des Coteaux (fig. 1 ; Airvaux 2002 ; Delage op. cit. ; Airvaux, Brou et Primault op. cit.).

\section{3 - Corpus de l'étude}

Le corpus étudié attribué au Magdalénien moyen regroupe pour les sites de Lussac-les-Châteaux : une pierre inédite des Fadets, qui demeure la seule figure de cheval connue sur 169 pierres (fig. 2) ; trois plaquettes de calcite alors inédites du réseau Guy Martin (pl. $1 \mathrm{n}^{\circ} 12-13$; relevés inédits aimablement confiés par J. Airvaux) ; 60 pièces en calcaire de la grotte de La Marche dont 12 de la collection du Musée de l'Homme qui ont fait l'objet d'un nouveau relevé analytique (fig. 3 ; pl. 1 ; Pales et Tassin de Saint Péreuse 1981). À Antigny, une seule plaquette gravée figurative a été mise au jour au Taillis des Coteaux (fig. 4), dans une couche datée et à proximité d'une pointe de sagaie rapprochée des pointes de type Lussac-Angles (Primault et coll. 2010). Enfin, deux fragments de plancher stalagmitique provenant du Puits au Chaffaud, mis au jour dans les déblais des fouilles anciennes, ont été comptés dans ce corpus (fig. 5 ; Airvaux 2002 ; Gaussein 2014).

\section{2 - Méthodes d'analyse}

\section{1 - Sources}

Cette recherche s'est basée d'une part sur l'observation directe d'un ensemble de pièces (collection du Musée de l'Homme à Paris, et collections Péricard et Airvaux de La Marche, conservées au Musée Sainte-Croix), d'autre part sur les publications du Dr. Léon Pales (Pales et Tassin de St. Péreuse 1981), de Jean Airvaux (Airvaux 2002) et de Nicolas Mélard (Mélard 2006), ainsi que sur les relevés inédits du réseau Guy Martin, aimablement confiés par Jean Airvaux à l'occasion de mon mémoire de Master.

\section{2 - Relevé analytique}

Quinze pièces dont deux inédites ont donné lieu à une analyse macro et microscopique, couplant le relevé (calque sur photographie), la photographie, l'observation à la loupe binoculaire et des analyses microtopographiques (Mélard op. cit. ; Gaussein 2012, 2014). Ces différentes techniques ont permis la mise en évidence d'informations qui n'étaient pas toujours prises en compte par les premiers chercheurs (Pales et Tassin de Saint Péreuse op. cit.). II est ainsi possible d'estimer les possibles différences de lecture entre notre travail de relevé sur un échantillon de pièces et les relevés des autres pierres sur lesquels repose l'analyse. II a pu être noté que certains relevés anciens étaient parfois largement sélectifs, et que certains reliefs naturels pouvaient être relevés parmi les tracés gravés.

\section{3 - Approche stylistique}

Ainsi que précisé en introduction, la présente étude interroge le degré de variabilité stylistique admis dans le cadre d'un même ensemble culturel défini par l'archéologie, sur une échelle spatiale réduite. Une analyse stylistique a donc été mise en œuvre, combinée à une étude du rapport entre les caractéristiques du support et les styles qui y sont appliqués. II sera ainsi possible de déterminer si celui-ci a un rôle à jouer dans la variabilité et les constances stylistiques. Chaque pièce du corpus a donc été décrite selon une grille de 180 critères, qui s'attachent à la description : du support gravé (neuf critères), des figurations de chevaux (124 critères), de la relation figuresupport (cinq critères) et des conditions d'isolat ou d'association des représentations équines analysées aux autres thèmes représentés sur une même pièce (42 critères : thèmes présents sur chaque face et phénomènes de superposition). Les critères de description des figures elles-mêmes s'organisent par thèmes : le profil, la portion de l'animal représentée (en pied, tête seule, etc.), l'allure et le mouvement (statique, cabré, tendu vers le sol, marche, etc.), la présence de repentirs, la description des détails de la tête (forme de la crinière, de l'œil, etc.), des membres (nombre, position, forme des extrémités), du corps (forme du ventre, du dos, etc.), de la queue et enfin

(5) Le Taillis des Coteaux niv. EG-Ilg : $14630 \pm 75$ BP (Ly-3876 ; Primault et coll. 2010).

(6) Le Puits du Chaffaud $15160 \pm 150$ B.P., niv. VII, faciès à navettes (Gif 735715 ; Airvaux 2001). 
Charte graphique des relevés : Relief et état du support

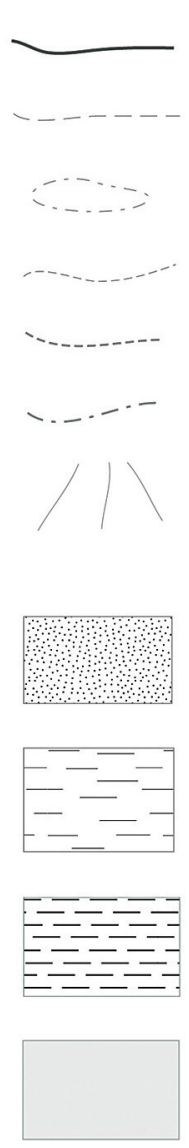

Limite fragments

Fissure

Dépression naturelle

Arrête de micro-relief

Arrête de relief marqué

Relief émoussé

Fracture conchoïdale

Encroûtement, concrétion

Zone faiblement altérée

Zone fortement altérée

Zone de clivage
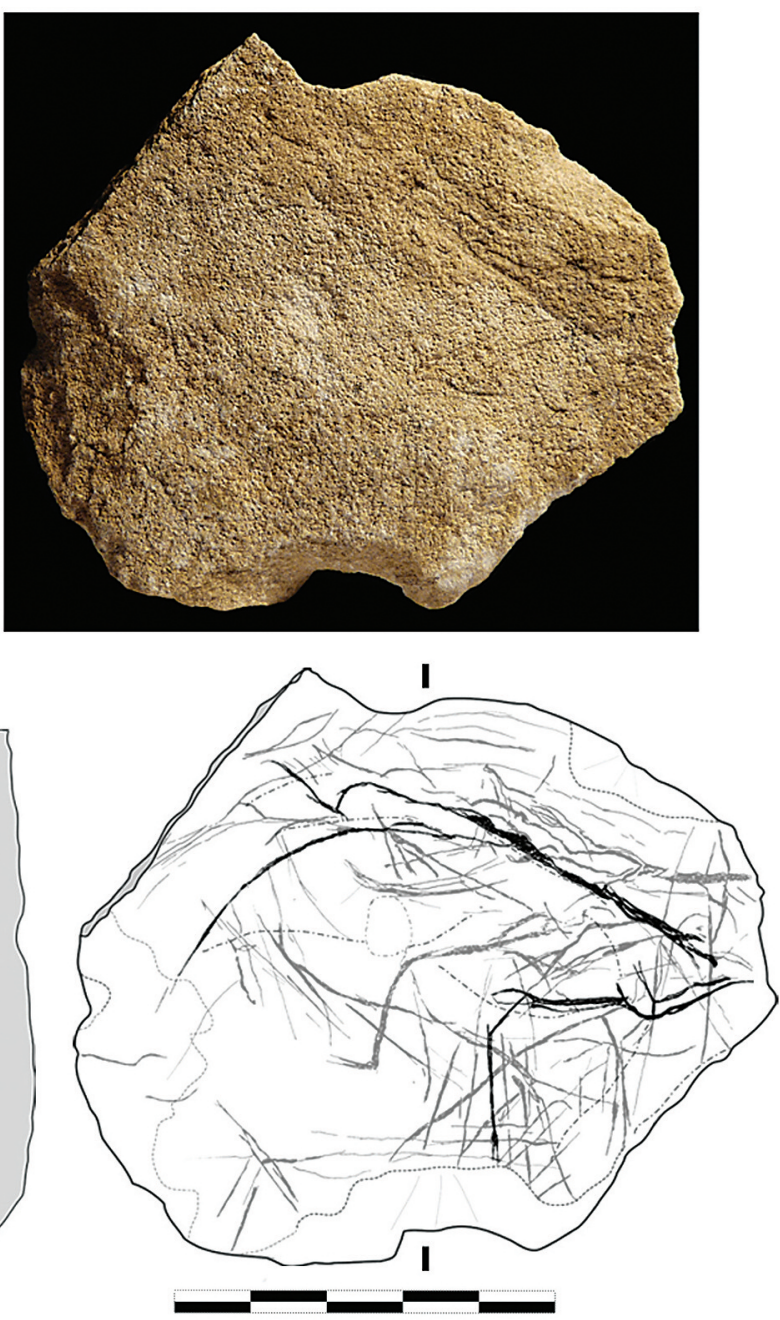

Figure 2 - Charte graphique des relevés : caractérisation du support ; Éclat de roche calcaire F. 63, grotte des Fadets (Lussac-lesChâteaux, Vienne). Musée de Préhistoire de Lussac-les-Châteaux (Les Sablines). Photographie et relevé P. Gaussein (2012).

Figure 2 - Graphic key for the drawings : characterization of the medium ; Limestone sliver F. 63, the Fadets cave (Lussac-les-Châteaux, Vienne). Prehistory museum of Lussac-les-Châteaux (Les Sablines). Photograph and drawing by P. Gaussein (2012).

la présence ou l'absence d'une ligne de sol. Ces multiples critères ont ensuite été soumis à des traitements statistiques simples (pourcentages, histogrammes et diagrammes circulaires).

Des notions sont mobilisées au cours de cette analyse, qu'il convient de définir en amont. Le «naturalisme » désigne le respect éthologique des représentations figuratives : allure, dynamisme, « scènes de vie » (abreuvement ou rut par exemple). Le « réalisme », quant à lui, fait ici référence au degré d'observation des formes, dimensions et détails des modèles vivants. La notion de bonne ou de mauvaise « facture » combine différents critères, à la fois stylistiques et techniques, qui ne reflètent pas des degrés de stylisation mais plutôt des indices de disparités de savoir-faire. La caractérisation d'une figure « maladroite » ou de « mauvaise facture » prend en compte une combinaison de critères : les difformités et disproportions (morphologie et morphométrie générale et des détails), le mauvais placement des formes et des détails en comparaison du modèle réaliste, la représentation rectiligne de segments de la figure normalement courbes, auxquels peuvent s'ajouter les rectifications multiples et autres signes de manque de maîtrise de l'outil de gravure et de son support (difficultés d'inciser des segments courbes d'un seul geste, dérapés de tracés notamment ; Rivero 2015). 


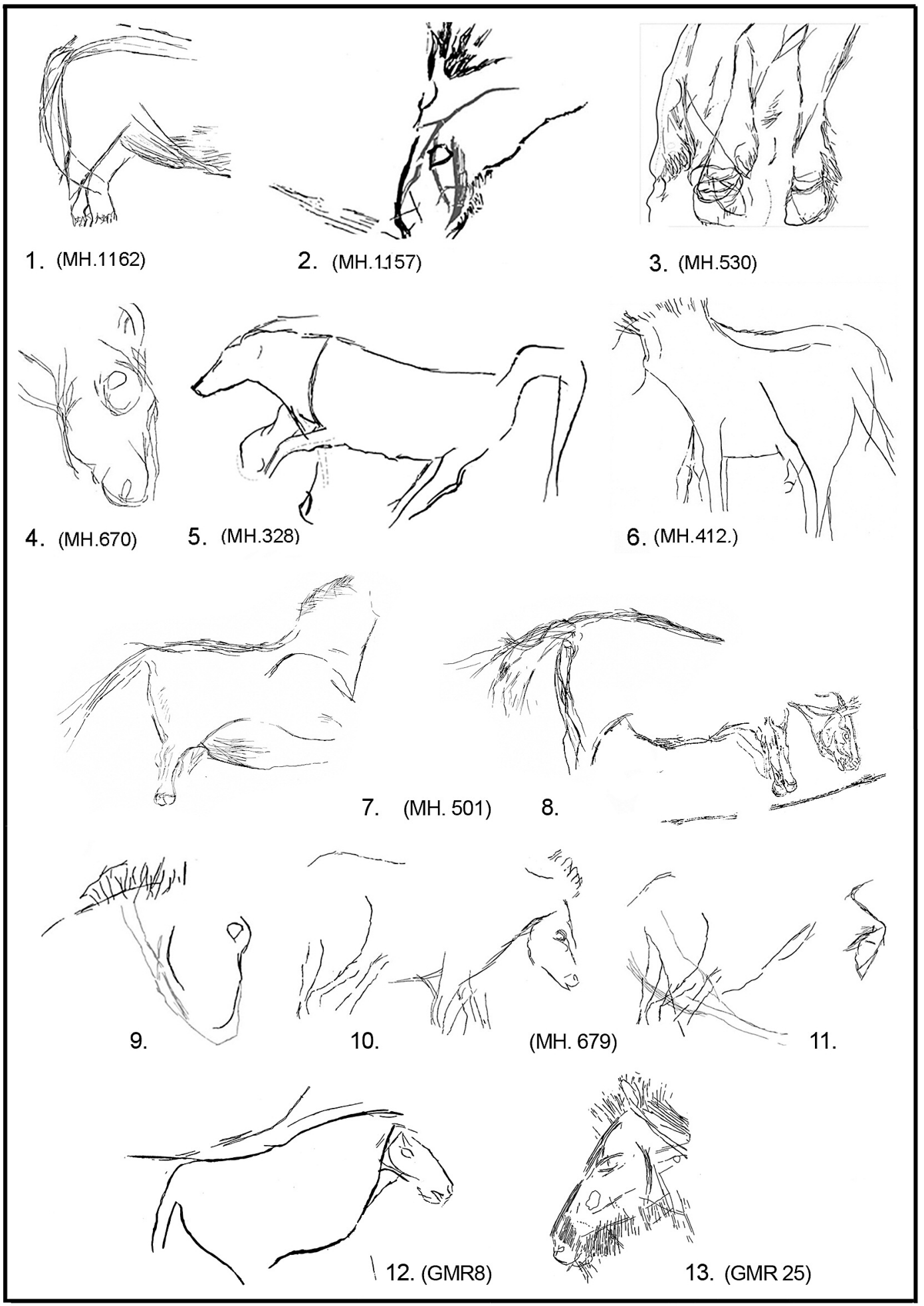

Planche 1 - Relevés sélectifs de chevaux des pierres gravées de la Vienne magdalénienne dans cette étude. 1. à 11. Pierres de La Marche (Lussac-les-Châteaux, Vienne) ; 12 and 13. Pierres du Réseau Guy Martin (Lussac-les-Châteaux, Vienne). 1-7 et 9-11 relevés par P. Gaussein (2012), 8. P. Gaussein et avant-main d'après L. Pales (Pales et Tassin de Saint-Péreuse 1981), 12. et 13. J. Airvaux.

Plate 1 - Selective Drawings of horse figures on rocks from Magdalenian Vienne in this study. 1. to 11. Rocks from La Marche (Lussac-les-Châteaux, Vienne). 12 and 13. Rocks from the Réseau Guy Martin (Lussac-lesChâteaux, Vienne). 1. to 7. and 9. to 11. drawings by P. Gaussein (2012), 8. by P. Gaussein and front part after L. Pales (Pales et Tassin de Saint-Péreuse 1981), 12. and 13. by J. Airvaux. 


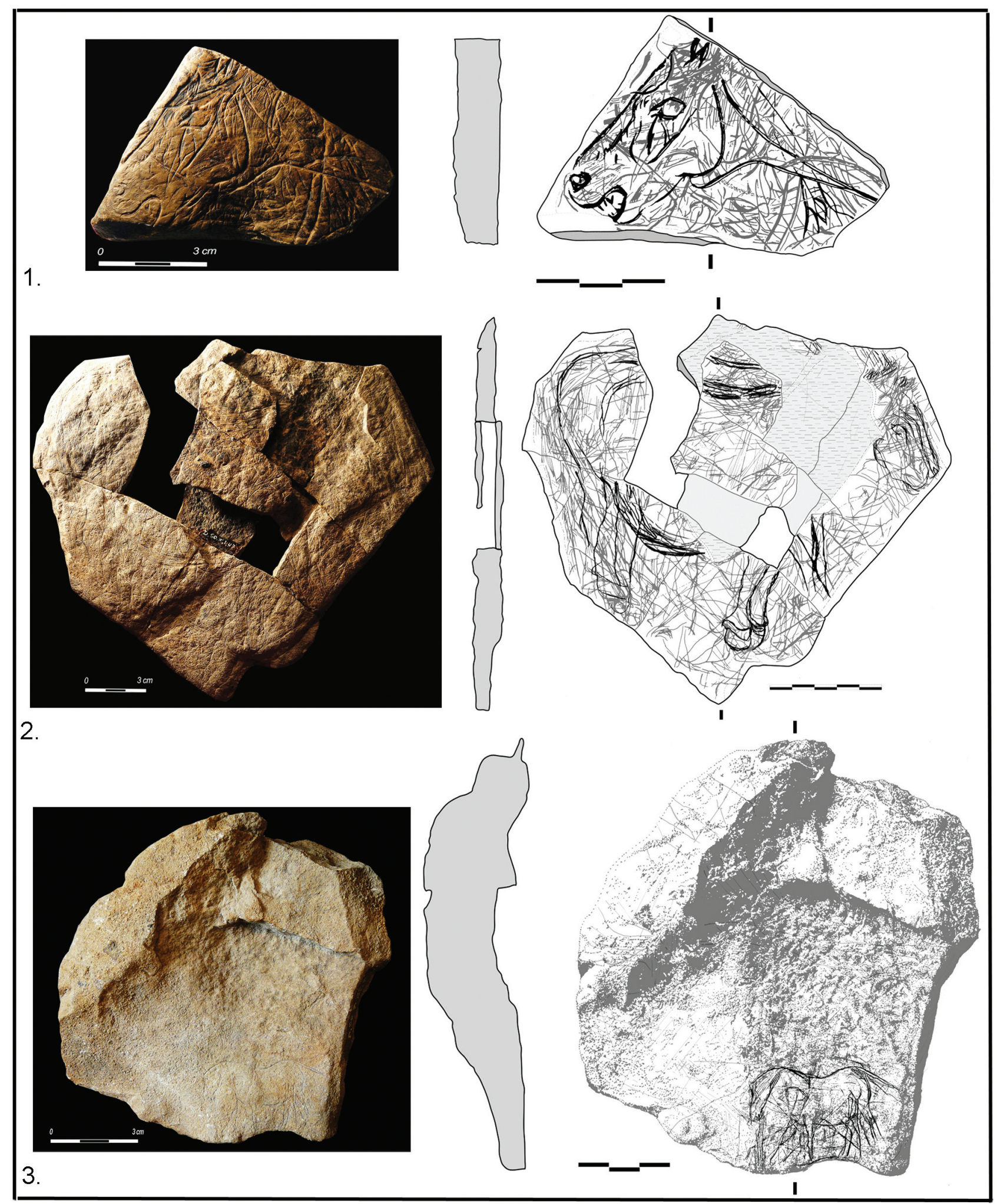

Figure 3 - Plaquettes calcaires, grotte de La Marche (Lussac-les-Châteaux, Vienne). 1. MH. 26 ; 2. MH. 287 a. et b. 336, 338, 401, 446 a. b. c., 447, 456 ; 3. MH. 324. Musée de L'Homme, Paris. Photographies et relevés P. Gaussein (2012).

Figure 3 - Limestone plaquettes, La Marche cave (Lussac-les-Châteaux, Vienne). 1. MH. 26; 2. MH. 287 a. and b. 336, 338, 401, 446 a. b. c., 447, 456; 3. MH. 324. Musée de l'Homme, Paris. Photographs and drawings by P. Gaussein (2012). 

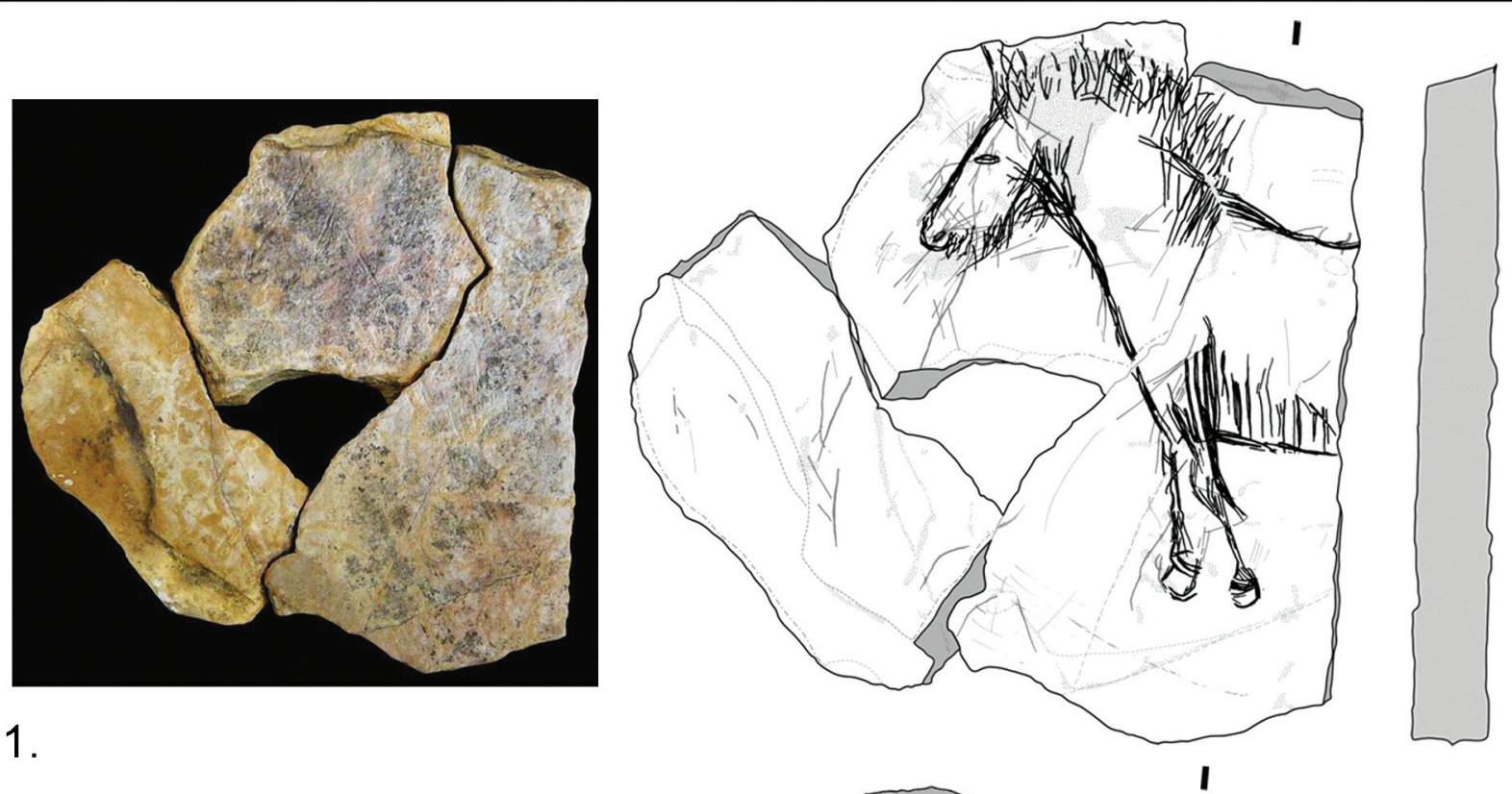

1.
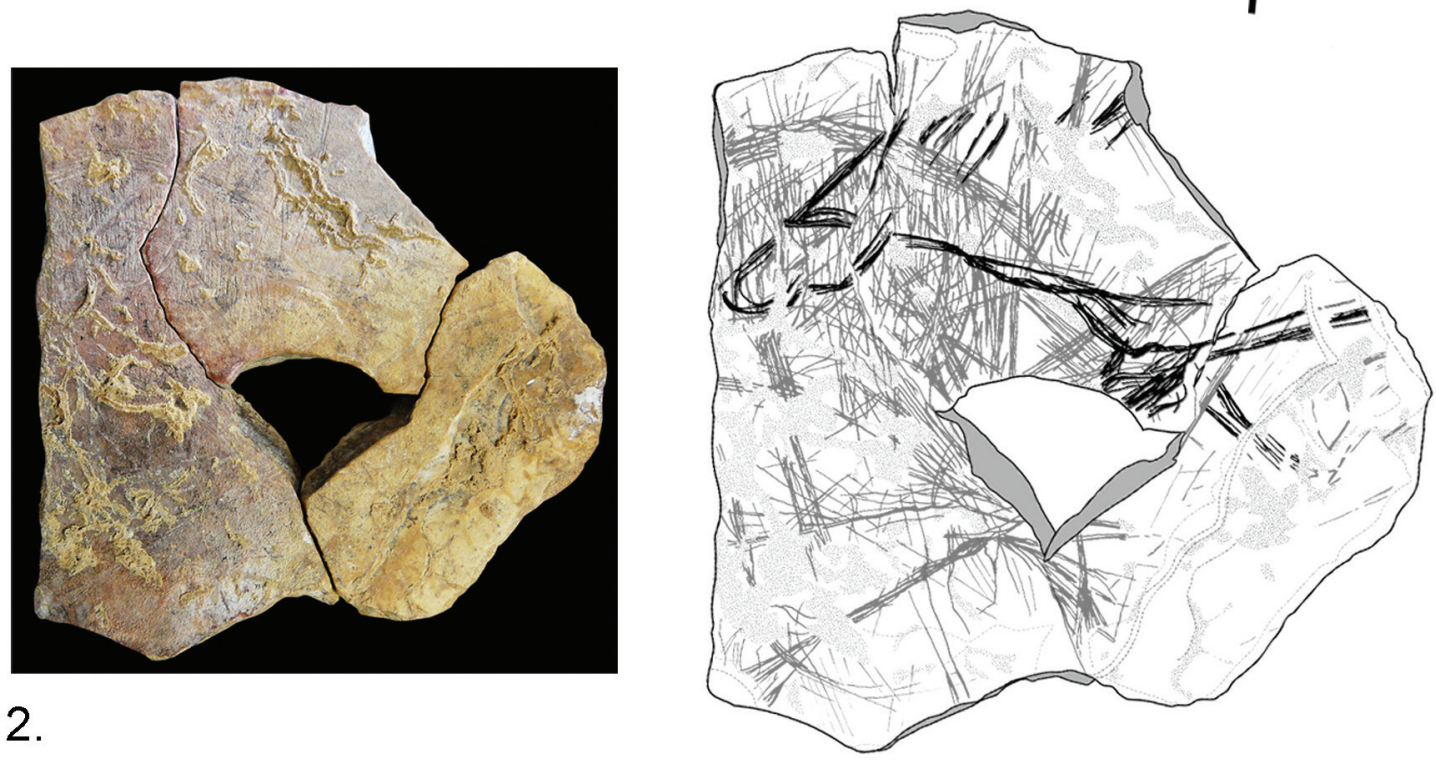

2.

Figure 4 - Plaquette calcaire (1.) recto et (2.) verso, Ilg, grotte du Taillis des Coteaux (Antigny, Vienne). SRA de Poitiers. Photographies et relevés P. Gaussein (2012).

Figure 4 - Limestone plaquette (1.) recto and (2.) verso, Ilg. Taillis des Coteaux cave (Antigny, Vienne). Archaeology Regional Service of Poitiers. Photographs and drawings by P. Gaussein (2012).

\section{3 - Observations :}

\section{supports et relations à l'image}

\section{1 - De la roche au support}

La matière première. Les différents étages géologiques et formations minérales définissent les dimensions des pierres, le type de surface, la couleur et la granulométrie des supports sélectionnés par les Magdaléniens (fig. 3-5). Les matières premières sont essentiellement locales. Les surfaces sont de qualité très variable : planes et lisses à irrégulières et rugueuses. Ce sont des pierres calcaires provenant de Lussac-les-Châteaux de qualité variable (La Marche, Les Fadets) et des fragments de plancher stalagmitique, aux surfaces lisses et ondulées, prélevés directement dans les grottes (réseau Guy Martin et le Chaffaud). Un calcaire kimméridjien exogène a été identifié au Taillis des Coteaux (fig. 4 ; Gaussein 2012), mais également à La Marche (Mélard op. cit.). II semble être un support idéal pour la gravure. Son origine a été définie selon un protocole comparable à l'analyse des gîtes de 


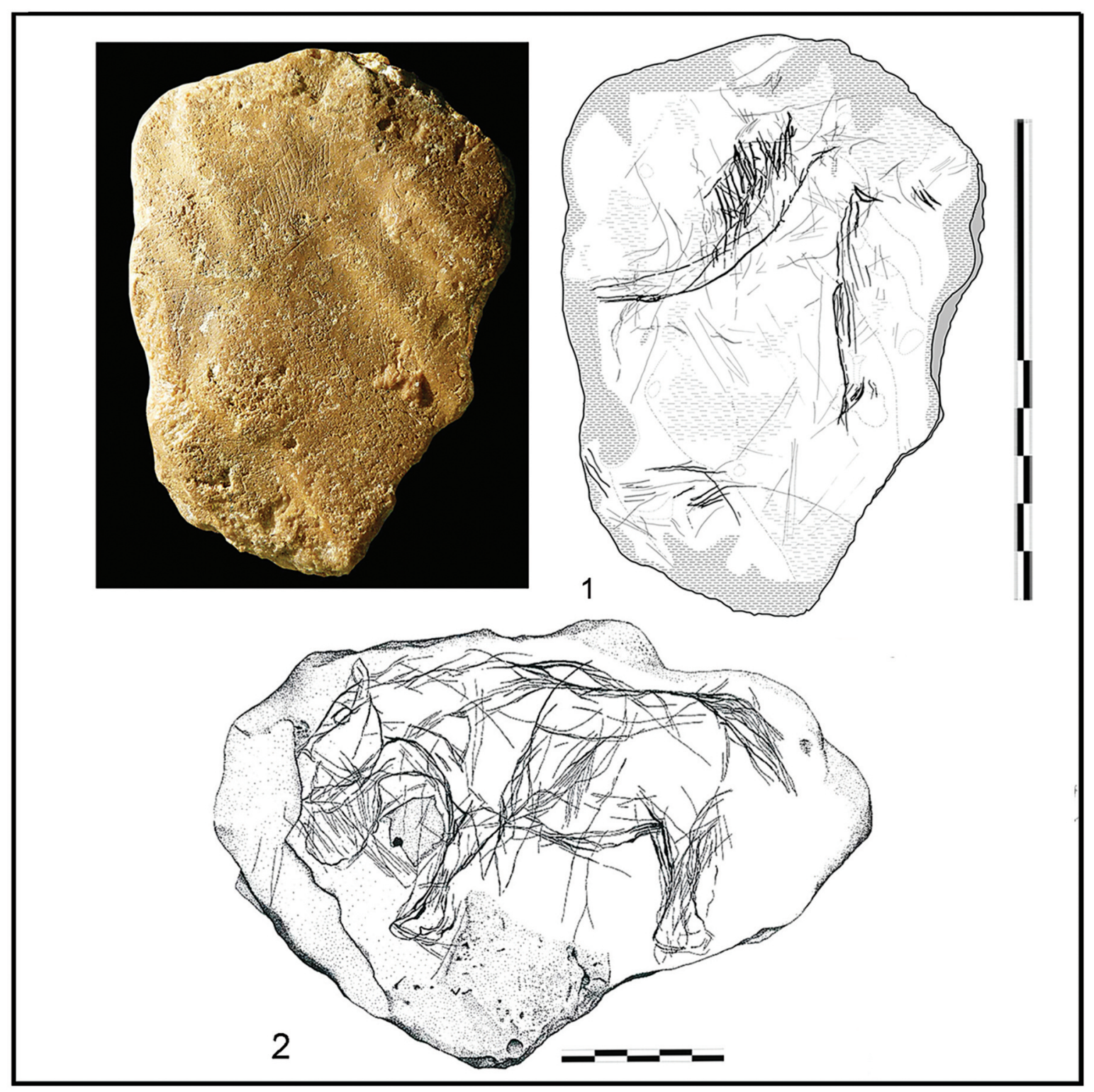

Figure 5 - Concrétions gravées, grotte du Chaffaud («grotte du Puits», Savigné, Vienne). 1. Petit bloc. SRA de Poitiers. Photographie et relevé P. Gaussein (2012, 2014) ; 2. Plaque. SRA de Poitiers. Relevé J. Airvaux (Airvaux 2002).

Figure 5 - Engraved speleothems, the Chaffaud cave («grotte du Puits», Savigné, Vienne). 1. Small block. Archaeological Regional Service of Poitiers. Photograph and drawing by P. Gaussein $(2012,2014)$; 2. Speleothem slab. Archaeological Regional Service of Poitiers. Drawing by J. Airvaux (Airvaux 2002).

\begin{tabular}{|c|c|c|c|c|}
\hline Support & Plaquette & Plaque & Dalle & Bloc \\
\hline Surface $(\mathrm{cm})$ & $<20$ & $\mathbf{2 0}$ à 50 & $>50$ & - \\
\hline Épaisseur $(\mathrm{cm})$ & $<5$ & $\begin{array}{c}<\text { à la moitié } \\
\text { de la } \\
\text { longueur }\end{array}$ & $\begin{array}{c}<\text { à la moitié } \\
\text { de la } \\
\text { longueur }\end{array}$ & $\begin{array}{c}>\text { ou }=\text { à moitié de } \\
\text { la longueur }\end{array}$ \\
\hline
\end{tabular}

Tableau 1 - Dimension des supports ornés et leur dénomination (d'après Tosello 2003).

Table 1 - Size and name for the engraved medium (after Tosello 2003). silex (Primault 2003) : une prospection sur le terrain orientée par une analyse des cartes géologiques de la France à $1 / 50000$ et de leur notice de caractérisation détaillée des faciès géologiques (BRGM), puis la comparaison à la loupe binoculaire des échantillons prélevés au cours de cette prospection et des pièces archéologiques concernées. Au final, les affleurements correspondant les plus proches sont situés à une cinquantaine de kilomètres au nord de la grotte, dans la banlieue de Châtellerault. 
Dimensions. La caractérisation des différents types de supports repose sur les critères définis par G. Tosello et sont reportés dans un tableau (tabl. 1 ; Tosello 2003). La moitié des supports lithiques utilisés est représentée par les plaquettes. Viennent ensuite les dalles $(26 \%)$ et les plaques (14\%). Blocs, éclats de roche et galets sont plus rares. La production de ces pierres est aisément expliquée par des processus naturels, notamment la gélifraction. Au contraire, les processus post-dépositionnels seuls ne suffisent pas pour expliquer la forte fragmentation postornementation de ces pierres : la quasi-totalité des pièces est incomplète, du simple coin de roche cassé aux pièces fragmentées en une dizaine de morceaux. Les techniques de fouilles anciennes et les aménagements des grottes au cours de l'Histoire ont pu y contribuer en partie, mais aussi des chocs plus anciens, naturels ou anthropiques, qu'ils soient accidentels ou volontaires (fonctionnels ou rituels, voir 5.1).

Traces diverses. Certaines pierres ont été exposées au feu après l'ornementation de l'une ou des deux faces (fig. 4). D'autres comportent de légères traces d'ocre rouge, ou de véritables points et plages de pigment (Mélard 2006 ; Gaussein 2012).

\section{2 - Le support et l'image}

La recherche d'effets visuels, élaborés à l'aide de différents types d'incisions et l'utilisation des reliefs et des formes naturelles, dépendent principalement de la nature du support. Dans un certain nombre de cas, les reliefs et les bords de la surface gravée peuvent participer à la représentation de la figure en créant un détail ou un léger modelé du corps et de la tête (fig. $3 n^{\circ} 1$ ), ou encore en constituant une ligne de sol (fig. $3 n^{\circ} 3$ et fig. $4 n^{\circ} 1$ ). Les calcaires lussacois y sont bien plus favorables que les surfaces planes et lisses du Kimméridgien ou encore que les ondulations des plaques stalagmitiques. Ainsi, certaines pierres pourraient avoir été sélectionnées, consciemment ou non, pour leurs formes et reliefs.

Dans certains cas, le support est littéralement intégré dans le processus de représentation d'une figure animale. En effet, nous avons pu observer des stigmates de modification de la surface gravée (fig. $6 n^{\circ} 1$ ) et des contours de deux pierres de La Marche qui ont fait l'objet d'un relevé analytique au cours de ce travail (fig. $6 n^{\circ} 2$ ).

La granulométrie et la dureté de la pierre ont pu influencer la finesse et le détail des figures. Naturellement, un support homogène au grain fin permet une exécution plus précise de la gravure (fig. $3 n^{\circ} 1$ ). Cependant, nous avons pu constater que des pierres de granulométrie moyenne $(0,5$ à $1 \mathrm{~mm})$ pouvaient être le support de figures de qualité très variable. Par exemple, le cas des miniatures de La Marche MH. 324 et Ja. 704 est remarquable (fig. 3 $n^{\circ} 3$ et fig. $7 n^{\circ} 2$ ). D'un autre côté, les surfaces idéales pour la gravure n'ont pas toujours été ornées de figures très

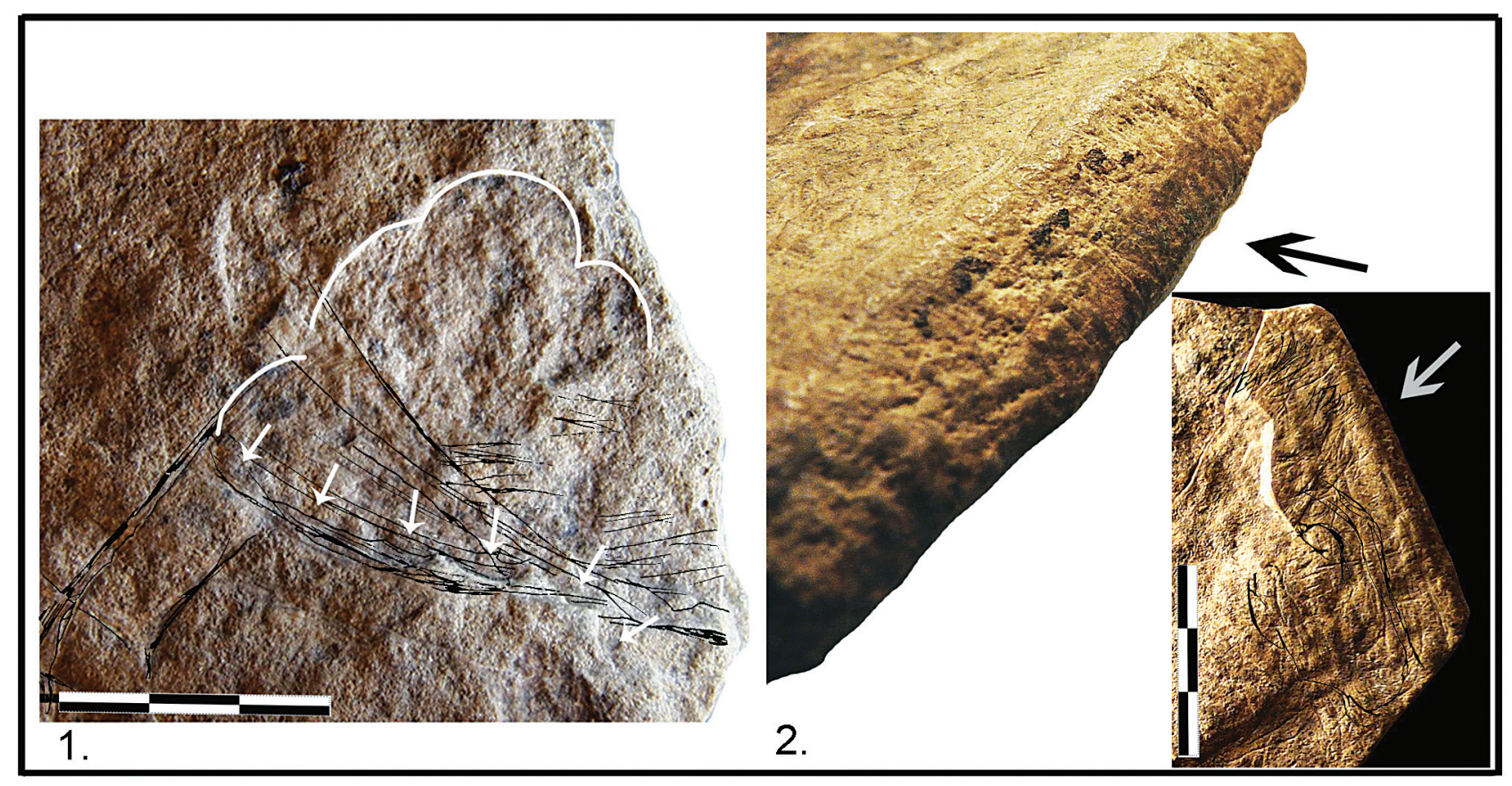

Figure 6 - Exemples de modification du support sur les pierres magdaléniennes de la Vienne. 1. Retrait d'un éclat de roche, plaquette MH. 1162. Arcs de cercle et flèches blanches en indiquent les bords ; 2. Abrasion du bord de la plaquette, MH. 287 et al. Musée de l'Homme, Paris. Photographies P. Gaussein (2012).

Figure 6 - Examples of modified medium on Magdalenian rocks from Vienne. 1. Removed rock sliver, plaquette MH. 1162. Arrows indicate the edges of the sliver; 2. Abraded edge of the stone, MH. 287 et al. Musée de l'Homme, Paris. Photographs P. Gaussein (2012). 


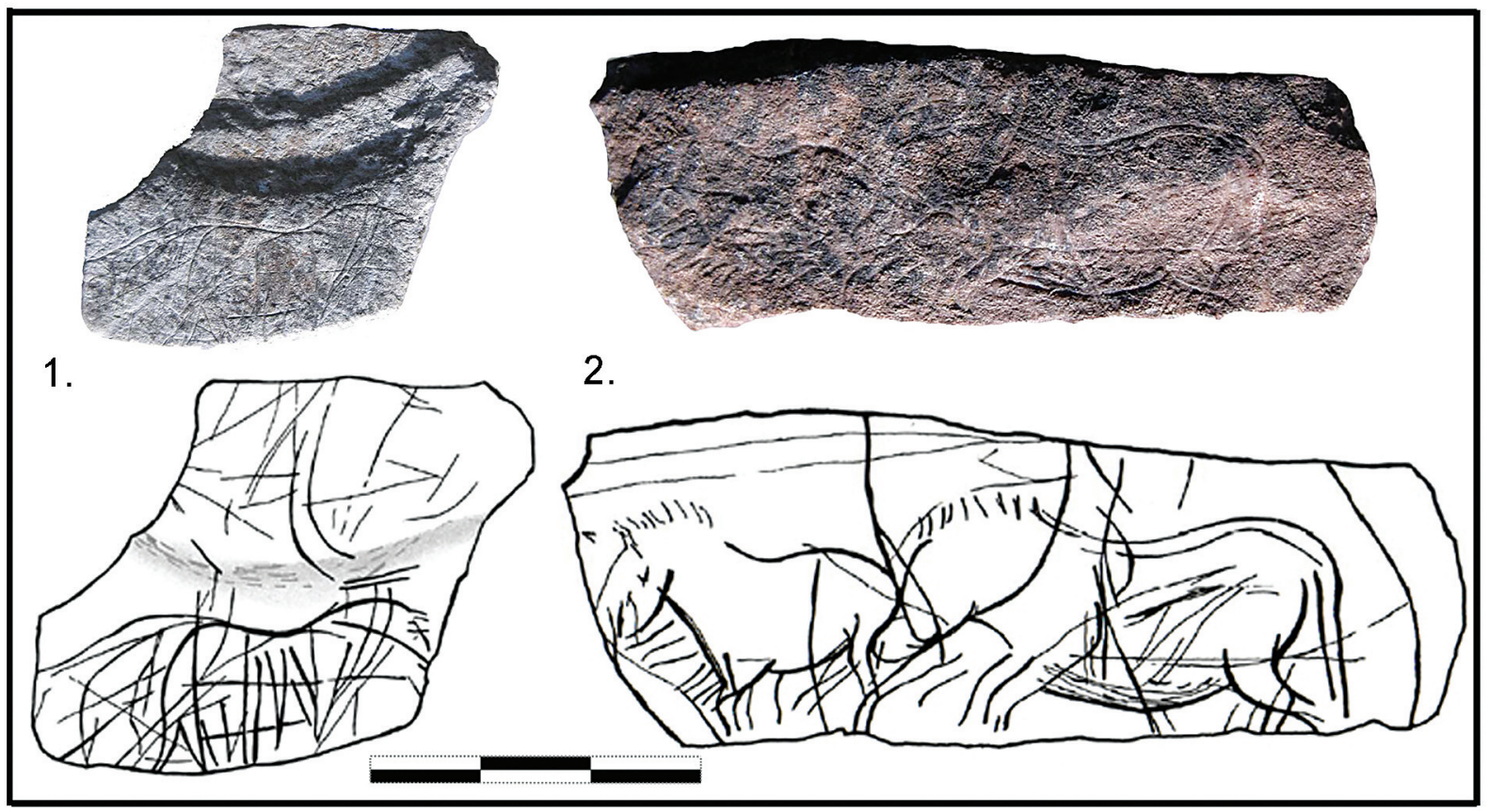

Figure 7 - Qualité du dessin selon la pierre sur les pierres magdaléniennes de la Vienne. 1. Plaquette lisse, Ja 712 ; 2. Plaquette rugueuse, Ja 704. Musée Sainte-Croix, Poitiers. Photographies et relevés N. Mélard (Mélard 2006).

Figure 7 - Quality of the drawing according to the rock on Magdalenian rocks from Vienne. 1. Smooth plaquette, Ja 712; 2. Ruguous plaquette, Ja 704. Sainte-Croix museum, Poitiers. Photographs and drawings by N. Mélard (Mélard 2006).

réalistes aux proportions exactes et aux détails nombreux et précis (fig. $4 n^{\circ} 2$ et fig. $7 n^{\circ} 1$ ).

Les supports choisis n'apparaissent donc pas contraignants au point de constituer un facteur majeur pour l'explication des variations et des constantes du style des représentations.

\section{4 - Observations : styles de représentation}

\section{1 - Isolements, associations, superpositions}

En grande majorité (53 pierres sur 67 ), les pièces sont gravées d'une ou deux figures (au moins un cheval). Dans la moitié des cas, un seul cheval a été représenté sur la pierre (les Fadets, Réseau Guy Martin, le Chaffaud). II est rare de trouver plus de quatre figures sur chaque pierre. Très souvent (79 figures sur 97, 61 pierres sur 67), les figures sont recoupées ou complètement superposées à d'autres unités graphiques, qu'elles soient figuratives (40 chevaux, 23 pierres) ou de simples tracés indéterminés (81 chevaux, 63 pierres ; voir fig. $3 n^{\circ} 1-2$ et fig. $4 n^{\circ} 2$ ). Les autres thèmes animaliers représentés sur les mêmes pierres que les chevaux sont principalement les cervidés (exclusivement des rennes lorsque la figure est identifiable) et les silhouettes animales d'espèce indéterminée (fig. 8 $n^{\circ} 1$ ). II faut noter la présence d'anthropomorphes (surtout sur la face opposée) et de félins. Les mammouths, ours, bisons et bouquetins y sont rarement associés.
Concernant les motifs et signes représentés sur ces pierres, une dizaine de "crinières isolées » et un ensemble de tracés en " bouquet énigmatique » ont été identifiés à La Marche par les premiers analystes (Pales et Tassin de Saint Péreuse 1981). Notre relecture des pierres nous permet d'y ajouter des signes foliacés (fig. $8 \mathrm{n}^{\circ} 2$ ).

Des tracés indéterminés ont été relevés sur $97 \%$ des surfaces étudiées et près de la moitié des faces opposées. Leur densité est très variable, de quelques incisions isolées (accidentelles ?) aux véritables lacis qui compliquent la lecture de la pierre (fig. $3 n^{\circ} 2$ ).

\section{2 - La figure du cheval dans le détail}

Profil et complétude. Une légère dominance des profils droits a été notée (58 sur 97 figures), ainsi que l'absence de figures représentées de face. La majorité des animaux est en pied (60) ; 22 sont réduits à la tête ou au protomé. Sur les 14 avant-mains et arrière-mains observées, certaines ont pu être originellement des représentations en pied, tronquées par la fragmentation des supports. Les dimensions sont variables, la taille des figures tendant à augmenter avec la taille du support.

Le support et le sol. Les reliefs et les bords du support ont souvent été recherchés comme éléments structurant la représentation animale. Par exemple, la ligne de sol, suggérée par le support ou véritablement tracée, est bien présente au Magdalénien moyen (fig. $3 n^{\circ} 3$; fig. $4 n^{\circ} 1$ et pl. $\left.1 n^{\circ} 2,8\right)$. La fiabilité de ce critère chronostylistique qui, selon certains auteurs, permettait d'exclure la phase 


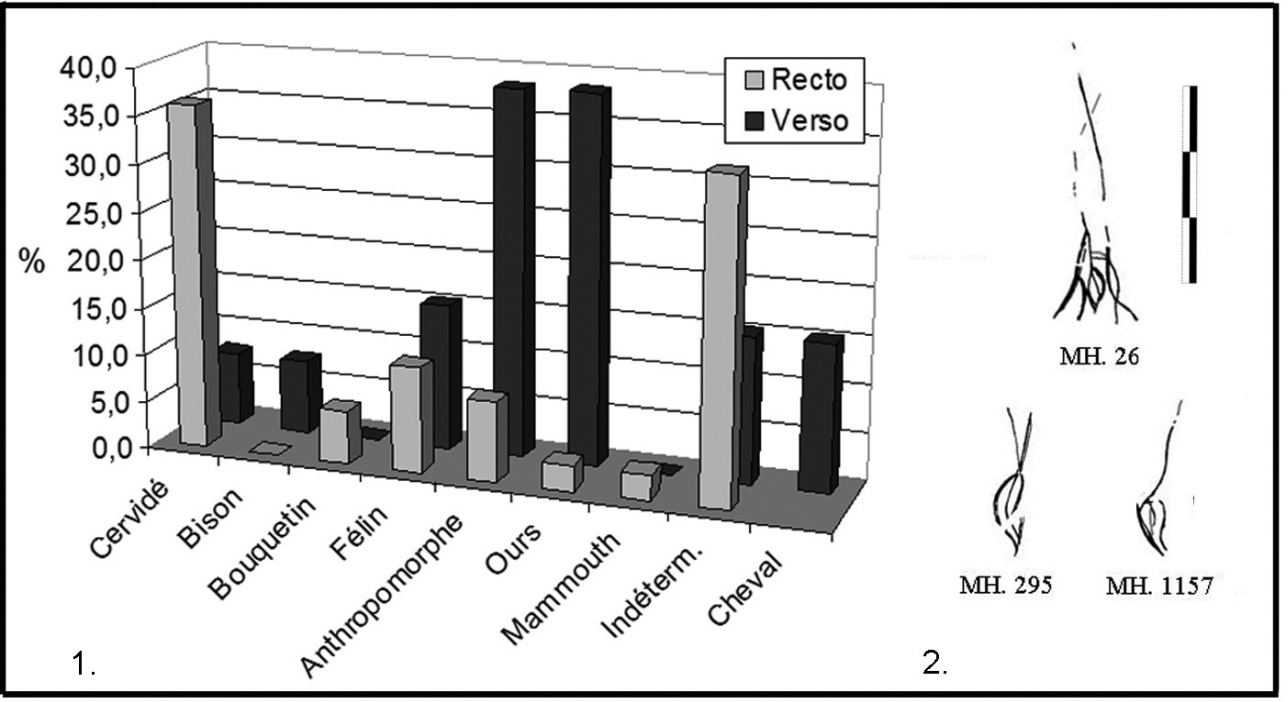

Figure 8 - Thèmes en association avec la figure du cheval sur les pierres magdaléniennes de la Vienne. 1. Thèmes représentés au recto et au verso ; 2. Signes foliacés identifiés sur les nouveaux relevés. Relevés $P$. Gaussein (2012).

Figure 8 - Themes

associated to the horse figure on Magdalenian rocks of the Vienne. 1. Animal figures represented on the recto and verso of the rocks; 2. Leaflike symbols identified on the new drawings. Drawings by P. Gaussein (2012).

moyenne du Magdalénien en faveur de sa phase récente (Airvaux et Leuvrey 2009) doit ainsi être révisée. Par ailleurs, il a été noté que les figures les plus réalistes ne sont pas nécessairement associées à une ligne de sol.

Naturalisme et mouvement. Les figures sont globalement dynamiques (55) et représentées dans des positions naturalistes. Souvent, la tête est tendue vers le sol (27 figures) et les membres sont en léger mouvement : cette posture évoque celle adoptée pour boire, manger ou pour effectuer le flehmen en période de rut ${ }^{7}$ (pl. $1 n^{\circ}$ 2-4, 8 et 10). D'autres sont plutôt représentés en pleine course (fig. $4 n^{\circ} 2$; pl. $1 n^{\circ}$ 5). Outre la tête et les membres, la queue emprunte également diverses positions, immobile ou en mouvement (fig. $3 n^{\circ} 3$; fig. $5 n^{\circ} 2$ et pl. $1 n^{\circ} 5-8,12$ ). Ces chevaux sont rarement complètement immobiles (11 cas) : les membres tendus à l'aplomb, la queue plaquée sur les fesses, l'axe de la tête formant un angle de $45^{\circ}$ avec l'encolure et regardant droit devant (Azéma 2006). La représentation de plusieurs versions d'une tête ou de membres est récurrente. Cet artifice semble servir cette recherche de dynamisme des figures gravées (fig. $3 n^{\circ} 3$; fig. $5 n^{\circ} 2$ et pl. $\left.1 n^{\circ} 6,10\right)$. Au contraire, les repentirs, rectifications de dessins, affectent plutôt les grandes lignes de la figure, de l'encolure à la croupe, le ventre et les lignes de la tête et du cou (fig. $3 n^{\circ} 2$; fig. $5 n^{\circ} 1$ et pl. $1 n^{\circ} 1,3$ ?, 11 ?).

Réalisme. Les détails essentiels de l'équidé sont généralement représentés. La crinière en séquence de tracés parallèles, sans ajout de ligne complémentaire, est la plus représentative statistiquement de la phase moyenne du Magdalénien. II existe cependant des variantes, plus rares dans ce corpus : un trait continu simple (fig. $3 \mathrm{n}^{\circ} 3$ et pl. $1 \mathrm{n}^{\circ} 5$ ), deux séquences de tracés (fig. $5 \mathrm{n}^{\circ} 1$ ) ou encore des chevrons (pl. $1 n^{\circ} 7$ ). Les extrémités des membres sont toujours représentées. Les détails tels que le pelage sont plutôt dessinés au Taillis des Coteaux, à Guy Martin et au Chaffaud (fig. $4 n^{\circ} 1$; fig. 5 et pl. $1 n^{\circ} 13$ ). Sur les figures de La Marche, le pelage apparaît plus rare. II faut cependant demeurer prudent : cette observation peut être un biais de lecture des relevés anciens et/ou résulter de l'altération différentielle des surfaces gravées, comme il a pu être constaté à plusieurs reprises au cours de cette étude. D'autres types de détails ne sont observés qu'à La Marche: modelé géométrisé de la tête et du cou (fig. $3 n^{\circ} 1$ ), fines hachures du ventre et de la fesse (pl. $1 n^{\circ} 1,7$ ), ou encore les vues en perspective du poitrail (fig. $3 n^{\circ} 3$ ) ou des lignes de fesse (pl. $1 n^{\circ} 8$ ). Par ailleurs, il est difficile de sexuer les animaux représentés. Un ventre particulièrement lourd pourra suggérer la gravidité d'une jument (22 chevaux sur 56 figures concernées), mais ce critère demeure très subjectif. Nous n'avons reconnu que deux étalons sans équivoque à La Marche : un cheval ithyphallique (pl. $\left.1 n^{\circ} 6\right)$ et un jeune étalon (fig. $3 n^{\circ} 1$ ) identifiable à ses canines nettement pointues (crochets ; Pales et Tassin de SaintPéreuse 1981). Mis à part ces tendances, il faut cependant remarquer que dans un même site, voire sur une même pièce, les figures peuvent être très détaillées à très sommaires (fig. 4). La tête des Fadets ne se rapproche franchement d'aucun style de représentation, si ce n'est de certaines figures sommaires de La Marche. Les autres pierres gravées du site sont ornées de figures d'une meilleure facture et plus naturalistes.

La tête du réseau Guy Martin est intéressante (pl. $1 \mathrm{n}^{\circ} 13$ ) : son dessin s'éloigne des plaquettes de La Marche et rappelle plutôt la morphologie et les détails du recto du Taillis des Coteaux (fig. $4 \mathrm{n}^{\circ} 1$ ).

Le petit bloc inédit du Chaffaud ne s'apparente nettement à aucun cheval du même site. La tête et les membres absents rendent difficile cette tentative d'attribution

(7) Flehmen, aussi dit « muser » : retroussement de la lèvre supérieure pour détecter les phéromones. 
chronostylistique. L'importance accordée au pelage de l'animal nous permet cependant de le rapprocher des figures qui viennent d'être évoquées.

Par ailleurs et pour conclure, les figures les plus détaillées n'intègrent pas systématiquement les reliefs de la surface, mais il faut noter que ce fait dépend en partie des propriétés du support, en particulier la présence ou l'absence de reliefs.

Autres thèmes sur pierre. Lorsque nous nous intéressons aux autres thèmes animaliers représentés, nous nous apercevons que les grandes constantes dégagées chez les chevaux peuvent en partie être appliquées aux cervidés, caprinés et bovinés, voire aux félins et ursidés. À La Marche, tous les thèmes sont dynamiques et adoptent des allures naturalistes, spécifiques à chaque espèce. Cependant, la qualité graphique des figures est très variable : certaines sont très sommaires, d'autres sont d'un réalisme et d'un naturalisme exceptionnels. Les figures aux têtes ou aux membres multiples sont plus rares; le pelage est plus souvent représenté chez ces différents thèmes que chez le cheval. Finalement, il ne s'agirait donc pas d'un biais lié à la nature et à la conservation du support, ou encore d'un biais de lecture.

\section{5 - Synthèse et discussion}

\section{1 - Graver les pierres dans la Vienne au Magdalénien moyen}

Une production locale. Ces pierres gravées sont principalement locales, et ne semblent finalement avoir été que peu déplacées depuis leur lieu d'acquisition. Dans la quasi-totalité des cas étudiés, la pierre sélectionnée est de qualité variable et se trouve en abondance à proximité directe du site où elles ont été abandonnées : falaises de Lussac-les-Châteaux, concrétions des grottes. II existe quelques cas à part : les supports exogènes dont la source a été identifiée (gîte à $40 \mathrm{~km}$ au nord de Lussac-lesChâteaux) présentent des qualités idéales pour la gravure. Ils témoignent de déplacements d'individus avec ces pierres, déjà gravées ou non, et dont la finalité nous échappe : expédition spécialisée pour l'approvisionnement des plaquettes à graver? Une acquisition opportuniste et/ou intégrée au cours de déplacements entre Poitou, faluns de Touraine (acquisition de coquillages) et gîtes de silex turonien entre Le Grand-Pressigny et la vallée de la Creuse ? En l'état de la recherche il est impossible de trancher.

Des images éphémères. Les figures sont incisées dans un matériau non périssable, mais il semble pourtant que la pérennité de l'image n'ait pas été recherchée, puisqu'elle disparaît souvent sous de nouvelles figures ou sous des tracés « parasites ». La primauté du geste sur l'image finale est l'interprétation le plus souvent avancée pour expliquer ce type d'objet (Fritz et Pinçon 1989). Le processus de représentation de l'image aurait donc eu plus de sens que sa préservation. C'est ce que suggèrent les observations de N. Mélard : des traces d'ocre en quantités variables ont été relevées sur les surfaces gravées et au fond de certains tracés, aussi, une couche de pigment aurait ainsi pu être appliquée pour faciliter l'incision de chaque nouvelle figure sur les précédentes. Cette figure apparaît ainsi nettement sur le fond coloré, mais disparaîtra à son tour sous une nouvelle application de colorant, un principe rappelant celui de l' " ardoise magique » (Mélard 2006). À cela s'ajoutent l'exposition au feu, la fragmentation des pierres, et finalement l'abandon de ces pièces, généralement sur le même site d'acquisition. Ce sont des gestes auxquels les chercheurs ont prêté du sens, tels que des gestes rituels de destruction de l'image (Clottes 1986 ; Fritz et Pinçon 1989 ; Mélard op. cit.). Cependant, cette pratique, du moins ces traces, ne sont pas généralisables et nuancent cette hypothèse interprétative. Les traces de chauffe et la fragmentation peuvent également résulter de leur simple abandon et/ou réutilisation (cycliques ou définitifs) par le même groupe ou par des groupes différents : une réutilisation domestique dans la structuration des foyers ; des processus de gélifraction ou de piétinements (réutilisation en dallage »; Mélard op. cit. ; Bosinsky 2007). II est difficile de trancher en l'état de ces travaux.

Des traditions esthétiques. À ces habitudes de traitement du support et de l'image s'ajoutent des traditions de représentation des figures et notamment de celle du cheval. La plupart des équidés sont individualisés par l'ajout de détails, le mouvement d'un membre, de la queue et/ou de la tête. Les animaux sont représentés dans des allures naturalistes. Mais il existe aussi de nombreuses figures sommaires, « maladroites » (Capitan et Bouyssonie 1924 ; Clottes 1986), indépendamment de la qualité du support. Par ailleurs, l'utilisation des formes et des reliefs naturels est récurrente mais non généralisable. II existe des cas de modification intentionnelle du support, mais aussi des exemples de distorsion de la figure pour un cadrage adapté à la surface du support. Comme nous l'avons vu, les disparités stylistiques ne dépendent donc pas nécessairement des propriétés du support : en d'autres contextes, il a effectivement été observé qu'un changement de matériau, de technique ou de sujet n'entraînait pas un " changement notable dans la forme fondamentale " (Schapiro 1982, p. 39). Un autre facteur doit donc entrer en jeu pour expliquer ces variantes esthétiques locales, relativement contemporaines. Différentes sources de variabilité stylistique identifiées en anthropologie sociale et culturelle peuvent être suggérées : différents degrés de savoir-faire et/ou d'apprentissage entre les graveurs ; des micro-styles se développant au cours des générations (modifications graduelles résultant des imperfections de la transmission des styles) ; des influences variables provenant d'autres groupes avec lesquels les graveurs de la Vienne auraient été en interaction (De Boer 1990 ; Cuche 2010 ; Lycett 2013).

\section{4 - Une identité stylistique par-delà les différences ?}

Un style régional ? De fortes ressemblances thématiques et stylistiques ont été observées entre certaines représentations pariétales et mobilières du Magdalénien moyen de la région. Les gravures pariétales du réseau Guy- 


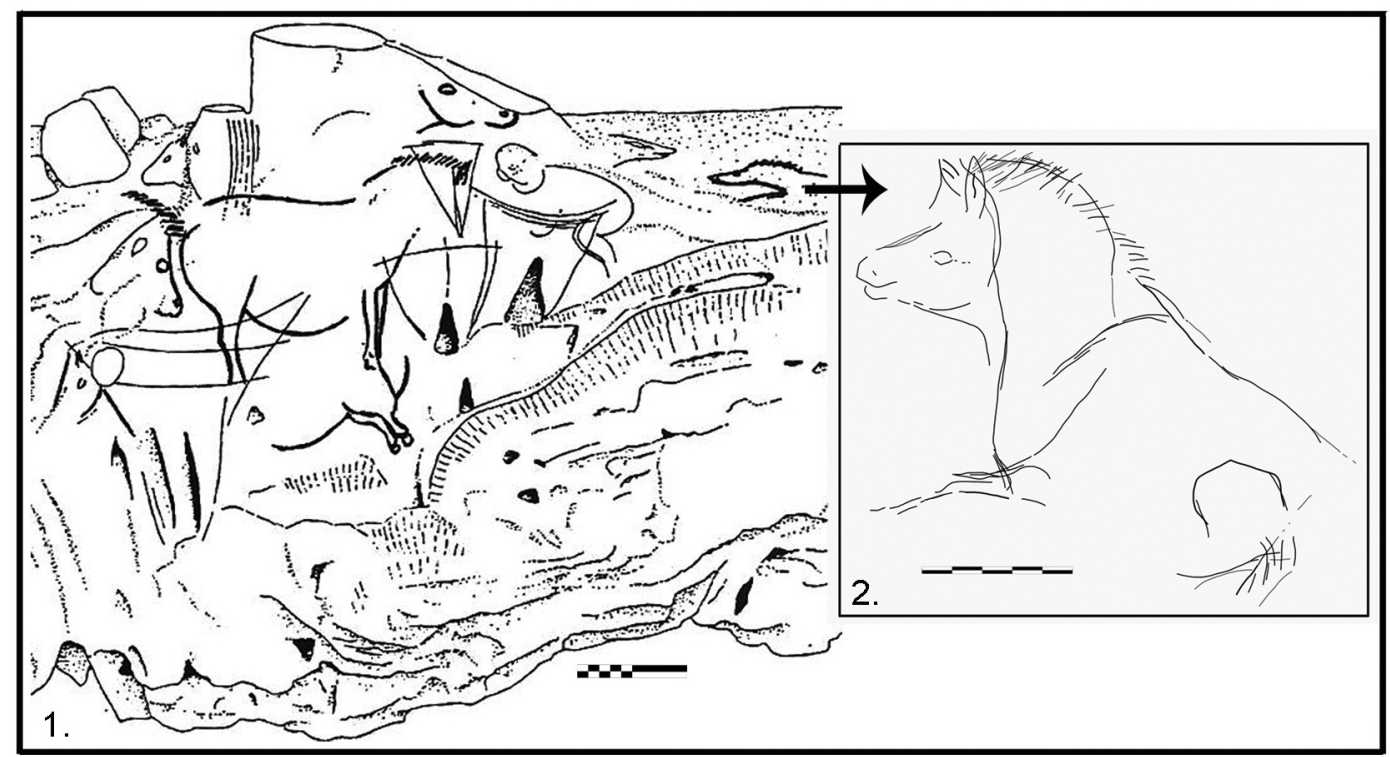

Figure 9 - Gravures pariétales du réseau Guy Martin (Lussac-les-Châteaux, Vienne). 1. Moitié droite du panneau sur coulée calcitique. Relevé général J. Airvaux (Airvaux 2001) ; 2. "Cheval bondissant» du coin supérieur droit du panneau. Relevé d'après J. Airvaux, musée Sainte-Croix, Poitiers.

Figure 9 - Engraved rock art from Guy Martin cave (Lussac-les-Châteaux, Vienne). 1. Right half of the speleothem panel. Global drawing J. Airvaux (Airvaux 2001); 2. "Jumping horse" from the right bottom corner of the panel. Drawing after J. Airvaux, Sainte-Croix museum, Poitiers.

Martin (Lussac-les-Châteaux) ne sont encore connues qu'à travers des relevés généraux préliminaires et un relevé détaillé exposé au musée Sainte-Croix à Poitiers (fig. $9, \mathrm{n}^{\circ} 1$; Airvaux 1998, 2001). Nous remarquons quelques similarités entre ces figures pariétales et les chevaux de l'art mobilier de La Marche, somme toute très classiques : la crinière hachurée et les formes naturalistes des membres, ainsi que le dynamisme du «cheval bondissant» (fig. 9, $\mathrm{n}^{\circ} 2$ ).

La frise de l'abri du Roc-aux-Sorciers à Angles-sur-l'Anglin est mieux documentée. Cet ensemble attribué au Magdalénien moyen fut exécuté en plusieurs étapes, correspondant à une évolution des thématiques représentées (lakovleva, Pinçon 1997 ; Pinçon [Ed.] 2009 ; Bourdier 2010b, 2013 ; Pinçon et al. 2013). Les figures d'équidés, tout comme les autres thèmes de ce site (principalement des bisons, humains, et bouquetins), sont exécutés dans un souci naturaliste et réaliste certain. Les formes, les proportions, les détails, ainsi que l'allure de ces représentations sont soignés. Les chevaux sont tous dynamiques, le mouvement étant suggéré par la multiplication des membres, ou la tête tendue vers le sol, les lèvres entrouvertes, dents apparentes, près des sabots antérieurs. Une forte parenté a ainsi été identifiée entre ces chevaux et les figures les plus soignées des pierres de La Marche, notamment en raison des détails énumérés à l'instant ainsi que pour la stylisation en triangle des reliefs de la tête (fig. 10 ; Bourdier 2010a, 2010b, 2013 ; Bourdier et al. 2016). Une première phase d'ornementation de l'abri, plus discrète (Pinçon et Bertrand op. cit. ; Pinçon [Ed.] op. cit.), s'apparente plus encore aux représentations des pierres gravées : sur le registre inférieur encore en place et dans les blocs détachés par les sculpteurs au Magdalénien moyen, des lacis d'incisions plus ou moins fines, indéterminées et figuratives, rappellent fortement les ensembles gravés des grottes de Lussac-les-Châteaux. Des contacts directs et une véritable contemporanéité semblent pouvoir être proposés pour certaines activités de ces deux occupations (Bourdier 2010b, 2013 ; Fuentes 2013a ; Bourdier et al. 2016 ; Fuentes et al. 2016).

Similarités inter-régionales. Des ressemblances de style, de manière de représenter certains détails figuratifs, sont à souligner entre les pierres de la Garenne ${ }^{8}$ et les pierres des vallées de la Vienne et de la Gartempe, ornées de tracés indéterminés et d'animaux au dessin plus ou moins « maladroit» (fig. 11). L'une de ces pierres s'apparente notamment à certains chevaux des plaquettes du Taillis des Coteaux (fig. 4) et du réseau Guy Martin (pl. $1 n^{\circ} 13$ ). La thématique animalière et la tendance stylistique des figurations seraient donc comparables, bien que ces occupations se distinguent par d'autres spécificités artistiques et technologiques (absence et présence de navettes et de sagaie de type "Lussac-Angles »). «Finalement, l'art mobilier de la « Garenne » est beaucoup

(8) La Garenne, datations des niveaux d'où proviennent les plaquettes de la fig. 11. Grotte Blanchard niv. B4 secteur HO : $15010 \pm 90$ BP (ETH-26100) ; Grand Abri niv. B2 secteur G : $15020 \pm 100$ BP (ETH-29158 ; Despriée et al. 2009). 


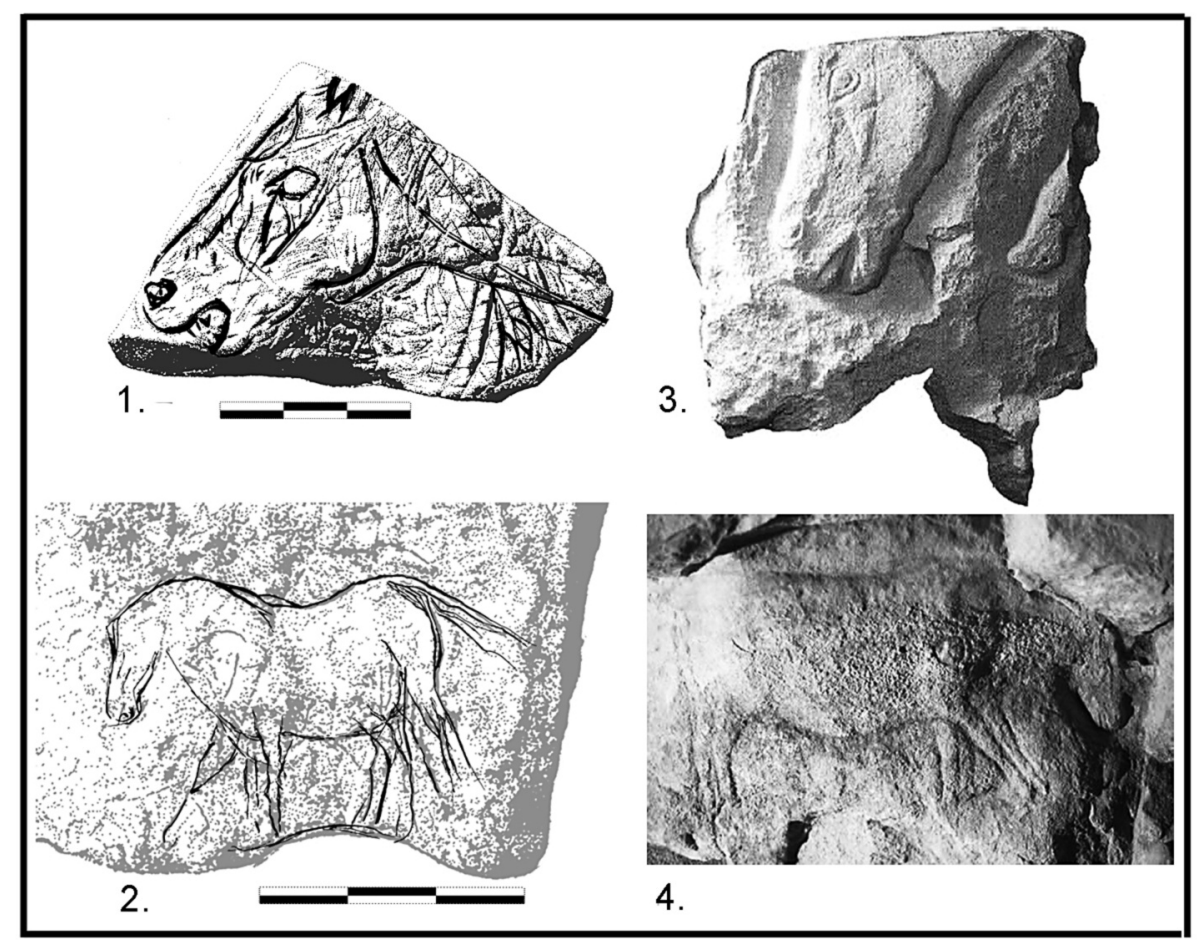

Figure 10 - Confrontation de figures du Magdalenien moyen de La Marche (1. et 2., Lussac-lesChâteaux, Vienne) et de l'abri du Roc-aux-Sorciers (3. et 4., Angles-sur-l'Anglin, Vienne). 1. Plaquette MH. 26, jeune étalon à la tête tendue, lèvres ouvertes, dents apparentes. Relevé P. Gaussein (2012) ; 2. Détail de la plaquette MH. 324, cheval en pied, la tête baissée, plusieurs membres représentés. Relevé P. Gaussein (2012) ; 3. Bloc de la frise BDD 168, cheval tête tendue, lèvres ouvertes, dents apparentes, MAN 83305 (43×39 cm). (lakovleva et Pinçon 1997) ; 4. "Cheval inclinant la tête » (Ch2), BDD 237 cheval en pied, la tête baissée, plusieurs membres représentés (70x56 cm). (lakovleva et Pinçon 1997).

Figure 10 - Comparison of Middle Magdalenian depictions from La Marche (1. and 2., Lussacles-Châteaux, Vienne) and from the Roc-aux-Sorciers rock shelter (3. and 4., Angles-surl'Anglin, Vienne). 1. Plaquette MH. 26, young stallion, head stretched forward, opened lips, visible teeth. Drawing by P. Gaussein (2012) ; 2. Detail from the plaquette MH. 324, full horse, lowered head, several legs represented. Drawing by P. Gaussein (2012) ; 3. Block BDD 168, head stretched forward, opened lips, visible teeth, MAN 83305 (43x39 cm). Photographie (lakovleva et Pinçon 1997) ; 4. "Cheval inclinant la tête " (Ch2), BDD 237, full horse, lowered head, several legs represented (70x56 cm). Photographie (lakovleva et Pinçon 1997).

plus conforme à ce que nous connaissons des productions artistiques dans le monde magdalénien. La présence d'un art sur plaquettes, même si elle est encore discrète pour l'instant, renvoie aux riches productions voisines du Magdalénien de «Lussac-Angles» [...] » (Paillet 2009 p. 198). La cohérence des faciès est d'ailleurs aujourd'hui interrogée et a fait l'objet de réflexions notamment au cours des Séances de la Société préhistorique française de Besançon (Bourdier et al. [Ed.] 2016), ainsi qu'au sein de deux programmes de recherche entre le sud-ouest de la France (MAGDATIS, dir. J.-M. Pétillon) et PoitouCharentes (dir. L. Brou).

Les productions graphiques du Magdalénien moyen des Pyrénées montrent également une certaine parenté (convergence ou traditions partagées ?) avec les figures du Centre-Ouest de la France : utilisation des reliefs du support, représentation du pelage, extrémité des membres figurée, traitement des fesses et du ventre par hachures (Fritz et Tosello 2001).

Des similitudes importantes ont également été repérées entre des figures du Poitou attribuées au Magdalénien moyen et des figures sur supports mobiliers et pariétaux d'attribution parfois incertaine au nord et à l'est de la Loire et dans le sud-ouest de la France. En Mayenne, les chevaux pariétaux de la grotte Margot (Thorigné-enCharnie, Mayenne) ressemblent fortement aux chevaux à la crinière et au pelage bien marqués du Taillis des Coteaux et du petit bloc inédit du Chaffaud (fig. $12 \mathrm{n}^{\circ} 1-5$ ). Ces figures finement gravées ont d'abord été attribuées au Magdalénien final (Pigeaud 2007b), puis ces chevaux à « crinière développée » ont plutôt été rapprochés du Solutréen (Pigeaud et al. 2012). Quoi qu'il en soit, des contacts entre les deux régions ont été identifiés à travers la circulation des matières siliceuses au cours du Magdalénien moyen (communication J. Primault). 


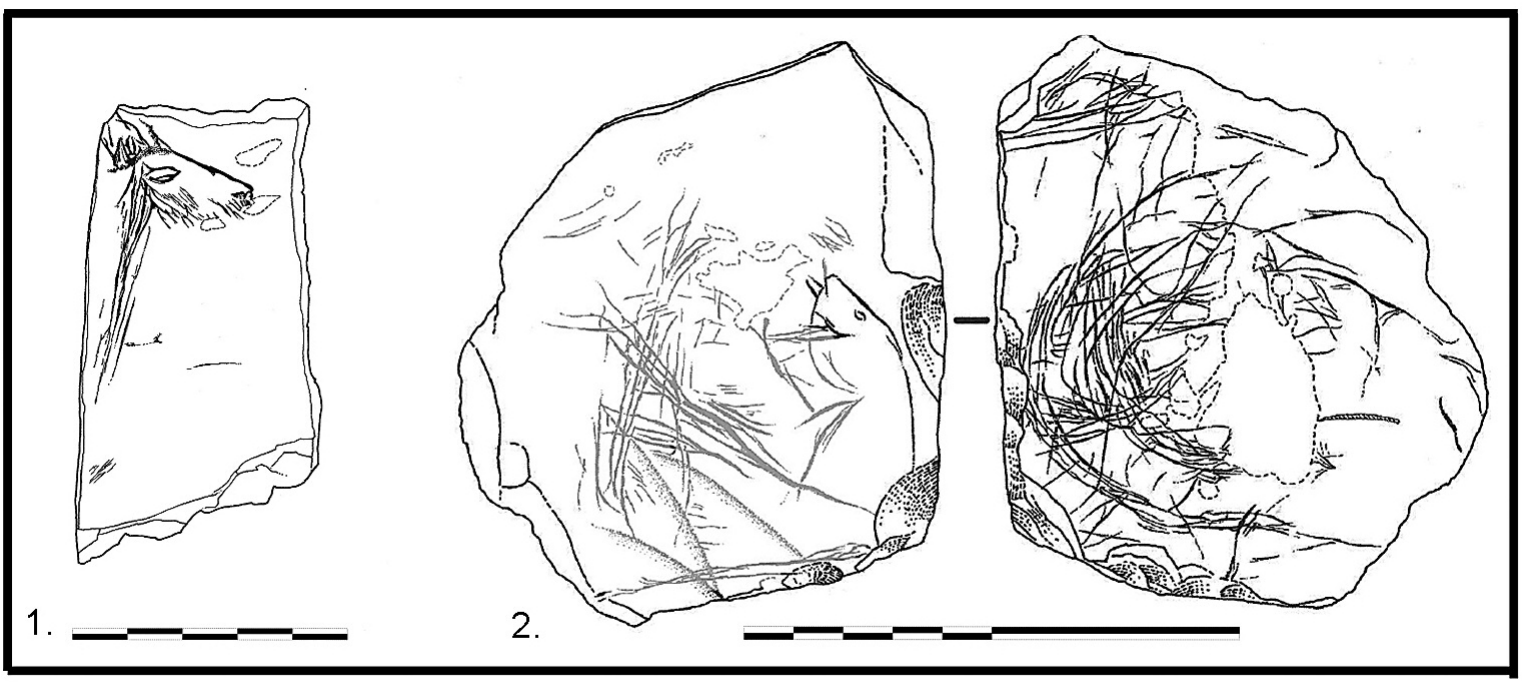

Figure 11 - Représentations de chevaux du Magdalénien moyen du site de La Garenne (Saint-Marcel, Indre). 1. Plaquette de schiste de la grotte Blanchard, 999-70-2, H0-B4 ; 2. Galet calcaire du Grand-Abri, 999-60-54, G-B2-90. Relevés P. Paillet (Paillet 2009).

Figure 11 - Horse depictions from Middle Magdalenian site La Garenne (Saint-Marcel, Indre). 1. Schist plaquette from the Blanchard cave, 999-70-2, H0-B4 ; 2. Limestone pebble from the Grand-Abri, 999-60-54, G-B2-90. Drawings by P. Paillet (Paillet 2009).

\section{6 - Conclusions}

Au travers de la maîtrise de la gravure, du choix des supports et de leur utilisation dans le processus de représentation du cheval, l'objectif de ce travail était d'explorer le degré de conventionnalité et de variabilité de l'art mobilier lithique dans une région et dans une phase donnée du Magdalénien.

D'une part, cette analyse a permis d'identifier de nouvelles figures et motifs, et de relever des détails qui ont échappé aux premiers chercheurs, ainsi que de mettre en évidence certaines faiblesses de l'outil chronostylistique : au sein de l'art magdalénien, la présence d'une ligne de sol gravée sous les membres des animaux n'est pas exclusive à sa phase récente.

D'autre part, la présence de détails, de pelage, de dynamisme des figures et de certaines postures naturelles redondantes, mais également la superposition des figures, de tracés indéterminés et les représentations " maladroites » caractérisent le corpus analysé. Aussi, malgré de fortes similarités entre certains sites, il a été observé une variabilité parfois importante des représentations figuratives sur les supports mobiliers lithiques, au sein d'une même subdivision d'un technocomplexe, d'un même faciès, et dans un espace d'expression restreint (même région, même site). Si elles n'entraînent pas une parfaite homogénéisation stylistique, ces pratiques entrent malgré tout dans une cohérence culturelle régionale et interrégionale.

Finalement, la variabilité stylistique - sur supports mobiliers lithiques - pourrait donc participer, en soi, à la caractérisation des pratiques artistiques du Seuil du Poitou au cours du Magdalénien moyen. Une forte identité symbolique, telle qu'observée par ailleurs dans l'art pariétal et en particulier dans les frises sculptées (Bourdier 2010a, 2010b, 2013), n'induirait donc pas nécessairement des conventions de représentations immuables, strictement normées, pour l'intégralité de la culture matérielle d'un ensemble identitaire.

Cette réflexion sur la portée des analyses stylistiques dans la définition de cultures et d'identités des populations préhistoriques s'est poursuivie dans le cadre d'un doctorat (2017, Université Paris Nanterre).

\section{Remerciements}

Nous remercions D. Vialou et P. Paillet pour leurs conseils. 


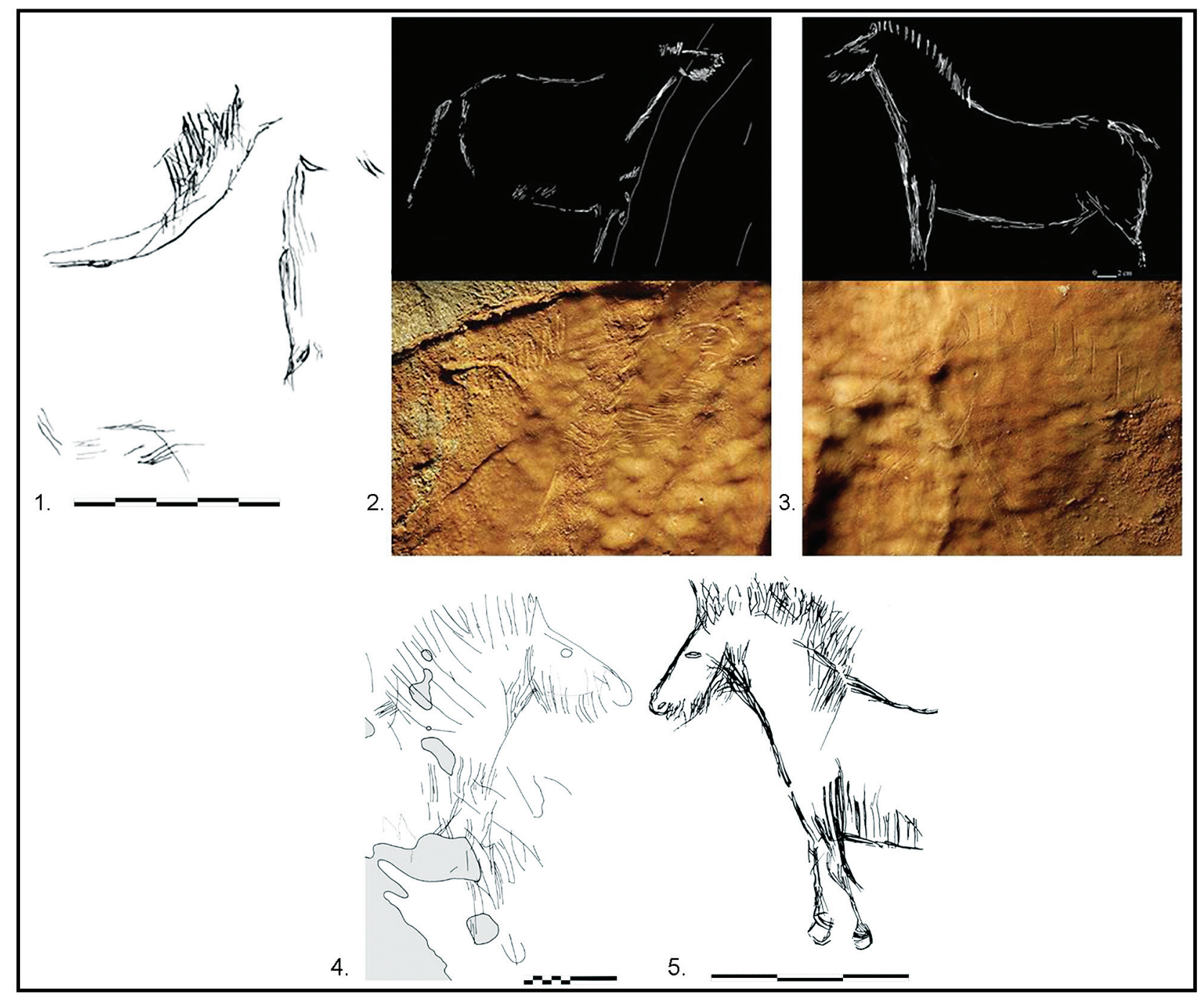

Figure 12 - Confrontation de figures du Magdalénien moyen de la Vienne (1. et 5.) et du Magdalénien récent du nord de la France (2.-4.). 1. Cheval sur bloc calcitique du Chaffaud (Savigné, Vienne). Relevé P. Gaussein (2012) ; 2., 3. et 4. Chevaux sur paroi de la grotte Margot (Thorigné-en-Charnie, Mayenne). Photographie des têtes H. Paitier, relevés P. Pigeaud, E. Tréholan et T. Devièse ; 5. Cheval du recto de la plaquette du Taillis des Coteaux (Antigny, Vienne). Relevé P. Gaussein (2012).

Figure 12 - Comparison of horse depictions from Middle Magdalenian Vienne (1. and 5.) and from Upper Magdalenian north France (2.-4.). 1. Horse on speleothem block from the Chaffaud (Savigné, Vienne). Drawing by P. Gaussein (2012) ; 2., 3. and 4. Rock art from the Margot cave (Thorigné-en-Charnie, Mayenne). Photographs of the heads H. Paitier, drawings by P. Pigeaud, E. Tréholan and T. Devièse ; 5. Horse on the upper face of the plaquette from the Taillis des Coteaux (Antigny, Vienne). Drawing by P. Gaussein (2012).

\section{Références bibliographiques}

AIRVAUX J. 1998 - Découverte d'une grotte ornée, le réseau Guy Martin à Lussac-les-Châteaux, Vienne, et application d'une méthodologie structurale pour l'étude de l'art préhistorique. L'Anthropologie, 102, 4, p. 495-521, fig.

AIRVAUX J. 2000 - Approche synthétique de l'art magdalénien de Lussac-Angles. In : P. Paillet (Ed.), Premiers artistes préhistoriques dans le Centre de la France. Exposition (30 juin-26 novembre 2000). Châteauroux, Musée d'Argentomagus, p. 47-61, fig.
AIRVAUX J. 2001 - L'art préhistorique du PoitouCharentes. Sculptures et gravures des temps glaciaires, la Maison des Roches éditeur, 223 p. ill.

AIRVAUX J. 2002 - Le Chaffaud, 168 ans après : À la mémoire de notre ami Henri Reigner qui participa aux recherches. Préhistoire du Sud-Ouest, 9, 1, p. 1-52, fig.

AIRVAUX J. 2006 - Les plaquettes calcaires du Taillis des Coteaux à Antigny (Vienne) : Inventaire du mobilier gravé et non gravé trouvé en 2006. In : J. Primault (Ed.), La grotte du Taillis des Coteaux, Antigny (Vienne), Fouille programmée triannuelle 2006 - 2008. Rapport d'activités 
2008, Service régional de l'archéologie Poitou-Charentes, p. 25-26, fig.

AIRVAUX J. 2011 - Les incisives de chevaux gravées du Magdalénien moyen de Lussac-Angles. Bulletins Préhistoire du Sud-Ouest, 19, 2, p. 137-195, fig.

AIRVAUX J. et LEUVREY J.-M. 2009 - Nouvelle découverte d'une œuvre de gravure à la grotte du Puits au Chaffaud à Savigné, Vienne. Préhistoire du Sud-Ouest, 17, 1, p. 115-125, fig.

AIRVAUX J., BROU L., PRIMAULT J. 2012 - Les outils sur lames tronquées et amincies du Magdalénien moyen de Lussac-Angles. Préhistoire du Sud-Ouest, 20, 2, p. 143178, fig. tabl. pl.

ALLAIN J., DESBROSSES R., KOZLOWSKI J.K., RIGAUD A., JEANNET $M$. et LEROI-GOURHAN A. 1985 - Le Magdalénien à navettes. Gallia Préhistoire, 28, 1, p. 37-124, fig.

AZÉMA M. 2006 - La représentation du mouvement au Paléolithique supérieur. Bulletin de la Société préhistorique française, $\mathrm{n}^{\circ} 103$, p. 479-505, fig. tabl. pl.

BOURDIER C. 2010a - Le Magdalénien moyen en PoitouCharentes. Une expression symbolique propre ?. In : J. Buisson-Catil et J. Primault (Eds.), Préhistoire entre Vienne et Charente. Hommes et sociétés du Paléolithique. Chauvigny : Association des Publications Chauvinoises, Mémoire XXXVIII, p.363-381, ill.

BOURDIER C. 2010b - Paléogéographie symbolique au Magdalénien moyen. Apport de l'étude des productions graphiques pariétales des abris occupés et sculptés de l'Ouest français. (Roc-aux-Sorciers, Chaire-à-Calvin, Reverdit, Cap-Blanc). Bordeaux : Université Bordeaux I, 2010. 2t., 403+223 p., ill. Thèse N.D. : Sc. : Bordeaux I ; 2.

BOURDIER C. 2013 - Rock art and social geography in the Upper Paleolithic. Contribution to the socio-cultural function of the Roc-aux-Sorciers rock-shelter (Angles-sur-l'Anglin, France) from the viewpoint of its sculpted frieze. Journal of Anthropological Archaeology, 32, 4, p. 368-382, fig.

BOURDIER C., CHEHMANA L., MALGARINI R. et POLTOWICZ-BOBAK M. (Ed.) 2016 - L'essor du Magdalénien. Aspects culturels, symboliques et techniques des faciès à Navettes et à Lussac-Angles. Actes des Séances de la Société préhistorique française $\mathrm{n}^{\circ} 8$ à Besançon (17-19 octobre 2013). Paris : Société préhistorique française, $260 \mathrm{p}$. fig.

BOURDIER C., BOSSELIN B., GAUSSEIN P., PAILLET P., PINÇON G. 2017 - Regards croisés sur la représentation animalière : choix thématiques et formels. In : C. Bourdier, L. Chehmana, R. Malgarini, M. PoltowiczBobak (Ed.), L'essor du Magdalénien : aspects culturels, symboliques et techniques des faciès à Navettes et à Lussac-Angles. Actes des Séances de la Société préhistorique française $n^{\circ} 8$ à Besançon (17-19 octobre
2013). Paris : Société préhistorique française, p. 103-117, fig.

CAPITAN L. et BOUYSSONIE J. 1924 - Un atelier d'art préhistorique. Limeuil. Son gisement à gravures sur pierres de l'Age du renne ?. Publications de l'Institut international d'Anthropologie, 1, Paris : E. Nourry, 41 p. ill.

CLOTTES J. 1986 - La détermination des figurations humaines et animales dans l'art paléolithique européen. In: T. Ingold et M. Maltby (Ed.), Cultural Attitudes to Animals Including Birds, Fish and Invertebrates, The World Archaeological Congress, 1-7 sept. 1986, Pré-Actes, Southampton et Londres, Allen \& Unwin, vol.3, p.1-29, fig.

CUCHE D. 2010 La notion de culture dans les sciences sociales. Paris : Découverte (4e édition), $128 \mathrm{p}$.

DE BOER W.R. 1990 Interaction, Imitation, and communication as expressed in style: the Ucayali experience. In: M. W. Conkey et C. A. Hastorf (Ed.), The Uses of Style in Archaeology. Cambridge, New York, Portchester, Melbourne, Sydney : Cambridge University Press, p. 82-104.

DELAGE C. 2011 - Le Site préhistorique de la Piscine (Montmorillon, Vienne). Vol. 1 Historique des recherches (1966 - 1982). Montmorillon : Les Éditions du Musée de Montmorillon, 47 p. III.

DESPRIÉE J., TYMULA S., RENAULT-MISKOVSKY J. 2009 - Chronologie absolue des sites magdaléniens du Coteau de "La Garenne » à Saint-Marcel (Indre). In : J. Despriée, S. Tymula et A. Rigaud (Ed.), Données récentes sur le Magdalénien de "La Garenne " (SaintMarcel, Indre). La place du Magdalénien «à navettes» en Europe. Actes du colloque d'Argenton-sur-Creuse (7-9 octobre 2004). Argenton-sur-Creuse, Bulletin de L'association pour la Sauvegarde du Site Archéologique d'Argentomagus et Amis du musée, ASSAAM n Spécial 2, p. 53-54, tabl.

DUJARDIN V. et PINÇON G. 2000 - Le Magdalénien dans la Vienne et la Charente. In: Pion G. (Ed.), Le Paléolithique supérieur récent : nouvelles données sur le peuplement et l'environnement, Actes de la table ronde de Chambéry, 1314 mars 1999, Mémoire XXVIII de la Société Préhistorique Française, p. 213-222, tabl.

FRITZ C. et PINÇON G. 1989 - L'art mobilier paléolithique. Valeur d'instants, de la création à la destruction. In : J.-P. Mohen (Ed), Le temps de la Préhistoire, t. 2 Interrogations sur l'art. XXIIle Congrès Préhistorique de France, Société Préhistorique Française, Arqueologia, p. 161-163, fig.

FRITZ C. et TOSELLO G. 2001 - Le cheval, acteur privilégié de l'art paléolithique. In : P. Brun (Ed), Le Cheval, symbole de pouvoir dans l'Europe préhistorique. Exposition, Nemours (31 mars-12 novembre 2001), musée de Préhistoire d'Île-de-France, p. 14-30, fig. 
FUENTES O. 2013a - La forme humaine dans l'art magdalénien et ses enjeux. Approche des structures élémentaires de notre image et son incidence dans l'univers symbolique et social des groupes paléolithiques. Thèse de Doctorat, Université Paris 1 Panthéon-Sorbonne, 2 vol. $718 p$.

FUENTES O. 2013b - The depiction of the individual in prehistory: human representations in Magdalenian societies. Antiquity, 87, p. 985-1000.

FUENTES O., LENOIR M., MARTINEZ M. et WELTÉ A.-C. 2016 - Les représentations humaines et leurs enjeux. Regards croisés entre le Roc-aux-Sorciers (Angles-surl'Anglin, Vienne) et le Roc-de-Marcamps (Prignac-deMarcamps, Gironde). In : C. Bourdier, L. Chehmana, R. Malgarini et M. Poltowicz-Bobak (Ed.), L'essor du Magdalénien. Aspects culturels, symboliques et techniques des faciès à Navettes et à Lussac-Angles. Actes des Séances de la Société préhistorique française $n^{\circ} 8$ à Besançon (17-19 octobre 2013). Paris : Société préhistorique française, p. 119-135, fig.

GAUSSEIN P. 2012 - Art gravé sur supports mobiliers lithiques de la Vienne magdalénienne. Analyses stylistique, technologique et tracéologique : la figure du cheval. Paris : Muséeum national d'histoire naturelle, 2012. 1 t., 210 p., ill. Mémoire de master 2 en Préhistoire : MNHN ; 1.

GAUSSEIN P. 2013 - De la liberté d'expression chez les Magdaléniens, Nouveaux éléments d'art mobilier de l'abri de La Piscine (Montmorillon, Vienne). Préhistoire du SudOuest, fig.

GAUSSEIN P. 2014 - Une nouvelle pierre gravée dans la grotte du Chaffaud (Savigné, Vienne). Bulletin de la Société Préhistorique Française, 111, 1, p. 139-142, fig.

IAKOVLEVA L., PINÇON G., 1997 - Angles-sur-l'Anglin (Vienne), La Frise sculptée du Roc-aux-Sorciers. Comité des Travaux Historiques et Scientifiques et Réunion des Musées Nationaux, Paris, $168 \mathrm{p}$.

LANGLAIS M. 2010 - Les Sociétés magdaléniennes de l'isthme pyrénéen, Ed. Cths, Comité Des Travaux Historiques et Scientifiques, 336 p. ill.

LANGLAIS M., SÉCHER A., CAUX S., DELVIGNE V., GOURC L. NORMAND C. et SÁNCHEZ DE LA TORRE M. 2015 - Lithic tool kits: A Metronome of the evolution of the Magdalenian in southwest France (19,000-14,000 cal BP). Quaternary International, p. 1-16.

LEROI-GOURHAN A. 1965 - Préhistoire de l'art occidental. Paris : Mazenod, 482 p. ill.

LYCETT S.J. 2013 - Cultural Transmission Theory and fossil Hominin Behaviour: A Discussion of Epistemological and Methodological Strengths. In: R. Ellen, S. J. Lycett, et S. E. Johns (Ed.), Understanding Cultural Transmission in Anthropology: A Critical Synthesis, Methodology \& History in Anthropology. New York/Oxford : Berghahn Books, p. $102-130$.
MÉLARD N. 2006 - Pierres gravées du Magdalénien moyen à La Marche, commune de Lussac-les-Châteaux (Vienne). Réalisation, fonction et interprétation. Paris : Muséum national d'histoire naturelle, 2006. 2t., 387 p., ill. Thèse de doctorat en Préhistoire : MNHN ; 2.

PAILLET P. 1998 - L'art paléolithique : tradition et modernité. Bulletin de la Société préhistorique française, 95,1 , p. 17-21, fig.

PAILLET P. 2009 - L'art mobilier sur supports lithiques de «La Garenne » (vallée moyenne de la Creuse, Indre). In : J. Despriée, S. Tymula et A. Rigaud (Ed.), Données récentes sur le Magdalénien de "La Garenne " (SaintMarcel, Indre). La place du Magdalénien "à navettes » en Europe. Actes du colloque d'Argenton-sur-Creuse (7-9 octobre 2004). Argenton-sur-Creuse : Bulletin de L'association pour la Sauvegarde du Site Archéologique d'Argentomagus et Amis du musée, ASSAAM n Spécial 2, p. 181-200, fig

PAILLET P., PINCON G. et BOURDIER C. 2016 Historique des recherches sur les faciès à Lussac-Angles et à navettes. In : C. Bourdier, L. Chehmana, R. Malgarini et M. Poltowicz-Bobak (Ed.), L'essor du Magdalénien. Aspects culturels, symboliques et techniques des faciès à Navettes et à Lussac-Angles. Actes des Séances de la Société préhistorique française $n^{\circ} 8$ à Besançon (1719 octobre 2013). Paris : Société préhistorique française, p. 119-135, fig.

PALES L. et TASSIN DE ST. PEREUSE M. 1981 - Les gravures de La Marche, III. Les équidés et bovidés. Paris : Ophrys, 145 p. ill.

PIGEAUD R. 2007a - Determining Style in Palaeolithic Cave Art: a New Method Derived from Horse Images. Antiquity, 81, 312, p. 409-422, fig.

PIGEAUD R. 2007b - Les chevaux des grottes ornées de la Mayenne. Equus, 70, p. 58-62, fig.

PIGEAUD R., HINGUANT S., PAITIER H., POMMIER V., BONIC P. et coll. 2012 - La grotte Margot (Thorigné-enCharnie, Mayenne) : un sanctuaire complexe aux influences multiples. Préhistoire, Art et Société, Ed. Société préhistorique Ariège-Pyrénées, LXVII, p. 81-101, fig.

PINÇON G. (Ed.) 2009 - Le Roc-aux-Sorciers : art et parure $d u$ Magdalénien. Réunion des Musées Nationaux (RMN), coll. Catalogues des collections, Paris ; disponible sur http://www.catalogue-roc-aux-sorciers.fr

PINÇON G., BERTRAND A. 2009 - L'industrie osseuse. In : G. Pinçon (Ed.), Le Roc-aux-Sorciers : art et parure du Magdalénien. Réunion des Musées Nationaux (RMN), coll. Catalogues des collections, Paris; disponible sur http://www.catalogue-roc-aux-sorciers.fr

PINÇON G., FUENTES O., ABGRALL A., BOURDIER C. 2013 - Pour une paléohistoire de l'image. Les jalons d'une réalisation iconographique : la frise magdalénienne du Rocaux-Sorciers (France), In : M. Groenen (ed.), Expressions 
esthétiques et comportements techniques au Paléolithique, Actes du XVle congrès de l'UISPP, Florianopolis, 4-10 sept. 2011, sessions 36 et 37, BAR International Series 2496, vol. 3, p. 55-72.

PRIMAULT J. 2003 - Exploitation et diffusion des silex de la région du Grand-Pressigny au Paléolithique. Nanterre : Université Paris Ouest, 2003. 1 t., 362 p., ill. Thèse de doctorat en Préhistoire : Paris X ; 1.

PRIMAULT J. 2012 - Circulations d'objets à grandes distances au Paléolithique. L'exemple de la diffusion des silex tourangeaux et poitevins. In : G. Marchand et G. Querré (Ed.), Roches et sociétés de la Préhistoire entre Massifs cristallins et bassins sédimentaires. Rennes, Presses Universitaires de Rennes, p. 81-92, fig. tabl.

PRIMAULT J. et collaborateurs 2010 - La grotte du Taillis des Coteaux à Antigny (Vienne). In : J. Buisson-Catil et J. Primault (Ed.), Préhistoire entre Vienne et Charente. Hommes et sociétés du Paléolithique, Chauvigny : Association des Publications Chauvinoises, Mémoire XXXVIII, p. 271-293, ill.
RIVERO O. 2015 - Art mobilier des chasseurs magdaléniens à la façade atlantique. Liège : ERAUL 146, 170 p., fig.

RIVERO O., SAUVET G. 2014 - Defining Magdalenian cultural groups in Franco-Cantabria by the formal analysis of portable artwork. Antiquity, 88, 339, p. 64-80, fig.

SCHAPIRO M. 1982 - Style, artiste et société. Paris : Gallimard, Bibliothèque des Sciences Humaines, 443p. ill.

TABORIN Y. 1993 - La parure en coquillage au Paléolithique. Supplément à Gallia Préhistoire, XXIX, Paris : CNRS, 531p.

TOSELLO G. 2003 - Pierres gravées du Périgord magdalénien, art, symboles, territoires. Supplément à Gallia Préhistoire, XXXVI, Paris : CNRS, 577p. ill. 